Article

\title{
Influence of Hard Segment Content and Diisocyanate Structure on the Transparency and Mechanical Properties of Poly(dimethylsiloxane)-Based Urea Elastomers for Biomedical Applications
}

\author{
Natascha Riehle ${ }^{1,2}$, Kiriaki Athanasopulu ${ }^{1,2}$, Larysa Kutuzova ${ }^{1,2}$, Tobias Götz ${ }^{3}$, Andreas Kandelbauer ${ }^{2}$ (D), \\ Günter E. M. Tovar ${ }^{3,4}$ and Günter Lorenz $2, *$ (D) \\ 1 Reutlingen Research Institute, Reutlingen University, Alteburgstr. 150, 72762 Reutlingen, Germany; \\ natascha.riehle@gmx.de (N.R.); kiriaki.athanasopulu@reutlingen-university.de (K.A.); \\ larysa.kutuzova@reutlingen-university.de (L.K.) \\ 2 Center for Process Analysis \& Technology (PA\&T), School of Applied Chemistry, Reutlingen University, \\ Alteburgstrasse 150, 72762 Reutlingen, Germany; andreas.kandelbauer@reutlingen-university.de \\ 3 Institute of Interfacial Process Engineering and Plasma Technology IGVP, University of Stuttgart, Nobelstr. 12, \\ 70569 Stuttgart, Germany; tobias.goetz@igvp.uni-stuttgart.de (T.G.); \\ guenter.tovar@igvp.uni-stuttgart.de (G.E.M.T.) \\ 4 Fraunhofer-Institute for Interfacial Engineering and Biotechnology IGB, Nobelstr. 12, \\ 70569 Stuttgart, Germany \\ * Correspondence: guenter.lorenz@reutlingen-university.de; Tel.: +49-7121-271-2027
}

Citation: Riehle, N.; Athanasopulu,

K.; Kutuzova, L.; Götz, T.;

Kandelbauer, A.; Tovar, G.E.M.;

Lorenz, G. Influence of Hard Segment

Content and Diisocyanate Structure

on the Transparency and Mechanical

Properties of Poly(dimethylsiloxane)Based Urea Elastomers for Biomedical Applications. Polymers 2021, 13, 212. https://doi.org/10.3390/

polym13020212

Received: 19 December 2020

Accepted: 4 January 2021

Published: 9 January 2021

Publisher's Note: MDPI stays neutral with regard to jurisdictional clai$\mathrm{ms}$ in published maps and institutional affiliations.

Copyright: () 2021 by the authors. Licensee MDPI, Basel, Switzerland. This article is an open access article distributed under the terms and conditions of the Creative Commons Attribution (CC BY) license (https:// creativecommons.org/licenses/by/ $4.0 /)$.
Abstract: The effect of hard segment content and diisocyanate structure on the transparency and mechanical properties of soft poly(dimethylsiloxane) (PDMS)-based urea elastomers (PSUs) was investigated. A series of PSU elastomers were synthesized from an aminopropyl-terminated PDMS $\left(\bar{M}_{n}\right.$ : $\left.16,300 \mathrm{~g} \cdot \mathrm{mol}^{-1}\right)$, which was prepared by ring chain equilibration of the monomers octamethylcyclotetrasiloxane $\left(\mathrm{D}_{4}\right)$ and 1,3-bis(3-aminopropyl)-tetramethyldisiloxane (APTMDS). The hard segments (HSs) comprised diisocyanates of different symmetry, i.e., 4,4'-methylenebis(cyclohexyl isocyanate) $\left(\mathrm{H}_{12} \mathrm{MDI}\right), 4,4^{\prime}$-methylenebis(phenyl isocyanate) (MDI), isophorone diisocyanate (IPDI), and trans1,4-cyclohexane diisocyanate (CHDI). The HS contents of the PSU elastomers based on $\mathrm{H}_{12} \mathrm{MDI}$ and IPDI were systematically varied between $5 \%$ and $20 \%$ by increasing the ratio of the diisocyanate and the chain extender APTMDS. PSU copolymers of very low urea HS contents (1.0-1.6\%) were prepared without the chain extender. All PSU elastomers and copolymers exhibited good elastomeric properties and displayed elongation at break values between $600 \%$ and $1100 \%$. The PSUs with HS contents below $10 \%$ were transparent and became increasingly translucent at HS contents of $15 \%$ and higher. The Young's modulus (YM) and ultimate tensile strength values of the elastomers increased linearly with increasing HS content. The YM values differed significantly among the PSU copolymers depending on the symmetry of the diisocyanate. The softest elastomer was that based on the asymmetric IPDI. The elastomers synthesized from $\mathrm{H}_{12} \mathrm{MDI}$ and MDI both exhibited an intermediate YM, while the stiffest elastomer, i.e., that comprising the symmetric CHDI, had a YM three-times higher than that prepared with IPDI. The PSUs were subjected to load-unload cycles at $100 \%$ and $300 \%$ strain to study the influence of HS morphology on 10-cycle hysteresis behavior. At $100 \%$ strain, the first-cycle hysteresis values of the IPDI- and $\mathrm{H}_{12} \mathrm{MDI}$-based elastomers first decreased to a minimum of approximately $9-10 \%$ at an HS content of $10 \%$ and increased again to $22-28 \%$ at an HS content of $20 \%$. A similar, though less pronounced, trend was observed at $300 \%$ strain. First-cycle hysteresis among the PSU copolymers at $100 \%$ strain was lowest in the case of CHDI and highest in the IPDI-based elastomer. However, this effect was reversed at $300 \%$ strain, with CHDI displaying the highest hysteresis in the first cycle. In vitro cytotoxicity tests performed using $\mathrm{HaCaT}$ cells did not show any adverse effects, revealing their potential suitability for biomedical applications. 
Keywords: segmented polyurea elastomers; poly(dimethylsiloxane); structure-property relationship; diisocyanate structure; transparency; mechanical properties; tensile hysteresis; in vitro cytotoxicity; biomedical applications; biomaterials

\section{Introduction}

Segmented polyurethanes (PUs) and polyureas (PUrs) are a broad class of polymers whose mechanical properties range from those of very soft elastomers to those of rigid thermoset-like materials with high mechanical strengths. This versatility is a result of the segmented structure of these polymers, which consist of alternating blocks of hard and soft segments. The soft segment determines the elastomeric properties and typically comprises macrodiols or diamines based on polyesters, polyethers, aliphatic polycarbonates, or, more recently, polybutadienes [1], polyisobutylenes [2], or poly(dimethylsiloxane)s with molecular weights between ca. 900 and $4000 \mathrm{~g} \cdot \mathrm{mol}^{-1}$ [3]. The hard segment (HS) consists of a diisocyanate and a low-molecular-weight diol or diamine chain extender and governs both the thermoplastic properties and the mechanical strength of the final polymer. Due to the inherent thermodynamic incompatibility of these segments, PUs and, in particular, PUrs display morphologies in which the HSs aggregate into hard domains that are phase separated from a surrounding matrix of soft domains [3].

The mechanical properties of PUs can be tailored according to required specifications by careful consideration of the chemistries, structures, and compositions of the macrodiol, diisocyanate, and chain extender moieties [4]. Therefore, PUs are widely applied in the fields of medical and biomedical engineering [5] for blood bags, tubing and valves, cardiac assist devices [6], drug-releasing coatings [7,8], antibacterial [9] and anti-biofilm coatings for urological catheters [10], and wound dressings [11].

The chemical stability of the soft segment is an important issue when considering the use of PUs in long-term implants like breast implant coatings [12] or artificial heart valves [13]. Polyester-based PUs degrade quite rapid in vivo as a result of hydrolytic cleavage of the ester bonds. For this reason, they are often utilized for short-term and biodegradable applications, e.g., scaffolds for meniscal reconstruction [14] or for the growth of human retinal epithelium cells after age-related macular degeneration (AMD) [15]. Conversely, poly(ether urethane)s, particularly PUs based on poly(tetramethylene oxide) (PTMO), are prone to oxidative degradation by environmental stress cracking and transition-metal-ion oxidation [16]. Therefore, poly(dimethylsiloxanes) (PDMSs), which are well known for their excellent biocompatibilities and chemical stabilities, are incorporated as co-macrodiols in polyether- and poly(carbonate urethane)s in order to enhance their biostabilities [17,18]. For instance, Gunatillake's group conducted systematic research on designing biostable PUs. They employed a mixed soft segment based on bishydroxyethoxypropyl PDMS along with the polyether poly(hexamethylene oxide) (PHMO) and successfully improved the biostability of the synthesized PU relative to a soft medical grade poly(ether urethane) [19]. Further research and development of these PUs led to their commercialization and approval as biomaterials for gastroenterological implants, lead insulators, and tri-leaflet heart valves [20].

An important factor for the design of mechanically stable PUs and PUrs is the composition and structure of the HS. PDMS-based PUs typically exhibit a high degree of microphase separation due to the strong hydrophobic nature of this macrodiol and the high polarity of the urethane groups [21]. However, most PDMS-based PUs lack sufficient mechanical stability, particularly when a high-molecular-weight PDMS is applied. However, Yilgör et al. showed that the synthesis of PDMS-based PUrs having reasonable mechanical properties, even at low HS ratios, is possible [22]. This difference in mechanical strength results from the strong bidentate intermolecular hydrogen bonding of the urea groups. Unlike real elastomers, which gain their mechanical strength from the chemical cross-linking of the polymer chains, the mechanical properties of PUs are largely dependent on the strength and 
effectiveness of their physical cross-links. Hence, the effects of the diisocyanate structure and symmetry on the morphology and tensile properties of PUs, polyurethaneureas, and PUrs has been addressed in various studies. For example, Adhikari et al. and Saralegi et al. both studied the influence of the symmetric trans-trans-4,4'-methylenebis(cyclohexyl isocyanate) $\left(\mathrm{H}_{12} \mathrm{MDI}\right)$ isomer ratio on the mechanical properties and microphase separation of PUs $[23,24]$. They found that microphase separation in the PUs increases due to a higher HS ordering. This led to an increase in Young's modulus (YM), hardness, and thermal stability. However, tensile strength seemed to be dependent on both the degree of HS crystallinity and the ability of the soft segment to form ordered structures upon strain [24]. Similarly, Joseph et al. studied the effect of varying the cis/trans-isomer distribution in 1,4-cyclohexane diisocyanate (CHDI)-based poly(ether urethanes) and also determined an increase in hardness and HS melting temperature when the ratio of trans-CHDI was increased [25]. HS crystallinity plays an important role in the mechanical properties of PUs, particularly on their tensile hysteresis behavior. This effect was thoroughly studied by Prisacariu et al. on a set of PUs based on 4,4'-methylenebis(phenyl isocyanate) (MDI), 4, $4^{\prime}$-dibenzyl diisocyanate (DBDI), and equimolar mixtures of these diisocyanates [26]. In contrast to MDI, in which the phenyl rings are linked by a methylene bridge, DBDI can adopt a more symmetric conformation due to rotation around the ethylene bridge, which promotes the formation of crystalline hard domains [27]. The stress-induced disruption of such domains was found to be accompanied by a higher hysteresis compared to that for HSs formed by MDI or diisocyanate mixtures. Morphological studies on non-chain-extended poly(ether urethane)s and PUrs containing diisocyanates of different symmetry in their HSs have been conducted, particularly by Yilgör and co-workers. They applied atomic force microscopy, small-angle X-ray scattering (SAXS), wide-angle X-ray scattering (WAXS), dynamic mechanical analysis, and tensile measurements to investigate the influence of hydrogen bonding and diisocyanate symmetry on microphase separation and morphology in these elastomers [28,29]. While the PUs, which contained the asymmetric diisocyanates $\mathrm{H}_{12} \mathrm{MDI}$, MDI, toluene diisocyanate (TDI), and $m$-phenylene diisocyanate ( $m$-PDI), were tacky, the analogous urea copolymers all displayed a microphase separated morphology and behaved as elastomeric materials. Furthermore, WAXS studies on urea copolymers revealed crystalline HSs except in those that contained isomeric mixtures $\left(\mathrm{H}_{12} \mathrm{MDI}\right.$, TDI). As a result, the stress in urea copolymers was higher compared to that in the urethanes. However, the first-cycle hysteresis values ( $300 \%$ strain) of the copolymers were generally very high (between $72 \%$ and $89 \%$ ). Interactions between HSs were also studied utilizing quantum mechanical calculations, dissipative particle dynamics (DPDs), and density functional theory (DFT) simulations [30,31]. The structures of model HSs as well as calculated hydrogen bond energies and distances revealed the presence of long-range-ordered hydrogen bonds in PUs and PUrs comprising symmetric diisocyanates (CHDI, HDI, $p$-PDI). This organization of hydrogen bonds allows tight packing of the chains and promotes the formation of crystalline domains, which corresponds to the reported results, such as high YM, tensile strength, and high hysteresis [30,31].

Most of these structure morphology relationship studies were performed on polyetherbased PUs and PUrs that were synthesized either without chain extenders or with symmetric chain extenders such as ethylene or butylene glycol. However, the etheric oxygen can interact with urethane/urea groups by hydrogen bonding, thus influencing morphology and mechanical properties [31]. Furthermore, the HS content and the soft segment molecular weight in the elastomers also have effects on mechanical properties [22].

Elucidation of the hard domain morphology was the main focus of the studies cited above. However, the resulting effect on tensile hysteresis behavior was often largely ignored in those investigations. Moreover, the optical appearance of the synthesized PUs was seldom addressed in this structure property context. Therefore, a study into both the influence of the diisocyanate structure and the effect of increasing the HS content on the mechanical properties and the transparency of PDMS-based urea elastomers is called for. 
Our previous study demonstrated that very soft aliphatic polysiloxane-based urea elastomers are obtained when the molecular weight of the PDMS soft segment is increased. These elastomers were intended for application as intraocular lenses and exhibit a transmission of approximately $90 \%$ between 750 and $500 \mathrm{~nm}$ until a PDMS molecular weight of $18,000 \mathrm{~g} \cdot \mathrm{mol}^{-1}$ [32]. The current work represents a significant extension of our earlier study published in Eur Poly. J [32]. In the present work, a number of different hard segment concentrations were studied, and, in contrast to the previous paper, several types of diisocyanates (see structures in Figure 1$)$ were investigated as well $\left(\mathrm{H}_{12} \mathrm{MDI}\right.$, IPDI, CHDI, MDI). Hence, the current work describes a much wider range of materials that were studied as potentially suitable biomaterials specifically for intraocular lens applications. For intraocular lens applications the optical properties of the materials are of special importance, in addition to the mechanical properties; therefore, the transparency of the produced materials and their biocompatibility were studied in detail.

This study presents the synthesis and characterization of a series of PDMS-based PUr elastomers (PSUs), which were prepared from an aminopropyl-terminated PDMS with a molecular weight of approximately $16,300 \mathrm{~g} \cdot \mathrm{mol}^{-1}$ and the diisocyanates $\mathrm{H}_{12} \mathrm{MDI}$, isophorone diisocyanate (IPDI), CHDI, and MDI. An aminopropyl-terminated disiloxane (APTMDS) was applied as a chain extender. To study the effect of the HS content on the transparency and mechanical properties of the PSU elastomers, the amount of HS was varied between $5 \%$ and $20 \%$ in aliphatic PSU elastomers prepared from $\mathrm{H}_{12} \mathrm{MDI}$ and IPDI. Furthermore, PSU copolymers were synthesized without the chain extender to investigate the influence of the diisocyanate structure on the mechanical properties of the PSU copolymers. The tensile hysteresis behavior of the PSU elastomers and copolymers was investigated in terms of their HS contents and the type of diisocyanate applied. For potential biomedical application of such PSU elastomers, e.g., in vascular prostheses, wound dressings, intraocular lenses, and soft coatings for medical devices, it is important that these materials do not show any adverse effects in contact with biological media and cells. Therefore, cell-medium extracts of the PSU elastomers were applied for in vitro cytotoxicity assessments to evaluate whether their low-molecular-weight leachables diminish cell viability.<smiles>O=[N+]([O-])C1CCC(CC2CCC([N+](=O)[O-])CC2)CC1</smiles><smiles>O=[N+]([O-])c1ccc(Cc2ccc([N+](=O)[O-])cc2)cc1</smiles><smiles>CC1(C)CC([N+](=O)[O-])CC(C)(CN=O)C1</smiles>

IPDI<smiles>O=NC1CCC([N+](=O)[O-])CC1</smiles>

Figure 1. Diisocyanates used in the synthesis of PDMS-based urea elastomers in this study: 4,4'-methylenebis(cyclohexyl isocyanate) $\left(\mathrm{H}_{12} \mathrm{MDI}\right), 4,4^{\prime}$-methylenebis(phenyl isocyanate) (MDI), isophorone diisocyanate (IPDI), and trans-1,4-cyclohexane diisocyanate (CHDI).

\section{Materials and Methods}

\subsection{Chemicals}

APTMDS (97\%) was purchased from ABCR GmbH (Karlsruhe, Germany). Tetrahydrofuran (THF) (anhydrous 99.8\%, stabilized with butylated hydroxytoluene) was purchased from Alfa Aesar (Thermo Fisher GmbH, Karlsruhe, Germany). MDI (98\%) was purchased from Sigma Aldrich Chemie GmbH (Taufkirchen, Germany). $\mathrm{H}_{12} \mathrm{MDI}$ and IPDI were kindly supplied by CSC Jäkle Chemie GmbH \& Co. KG (Nürnberg, Germany). CHDI was synthesized in-house according to a method published elsewhere [33]. The purities of the applied 
diisocyanates ( $\mathrm{H}_{12} \mathrm{MDI}$, IPDI, and CHDI) were at least $99 \%$ as determined by the dibutylamine back titration method carried out according to DIN EN ISO 14896:2009-07. $\alpha, \omega$-Bis (3-aminopropyl)-PDMSs with molecular weights of ca. $16,300 \mathrm{~g} \cdot \mathrm{mol}^{-1}$ (see Table 1) were prepared by ring-chain equilibration of octamethylcyclotetrasiloxane $\left(\mathrm{D}_{4}\right)$ and APTMDS according to a method described elsewhere [34].

Table 1. Molecular weights of the aminopropyl-terminated PDMSs used for synthesis of PSU elastomers in this study.

\begin{tabular}{|c|c|c|c|c|}
\hline PDMS $^{1}$ & $\begin{array}{c}\text { Molar Ratio } \\
\text { (D } \mathrm{D}_{4} \text { /APTMDS) }\end{array}$ & $\begin{array}{c}\text { Conversion } \\
(\%)\end{array}$ & $\begin{array}{c}\bar{M}_{n} \\
\text { Theoretical }^{2} \\
\left(\mathrm{~g} \cdot \mathrm{mol}^{-1}\right)\end{array}$ & $\begin{array}{c}\bar{M}_{n} \\
\text { Titration }^{3} \\
\text { (g. mol }{ }^{-1} \text { ) }\end{array}$ \\
\hline PDMS & $60.6 / 1.0$ & 88.8 & 16,175 & 16,338 \\
\hline PDMS (MDI) & $60.5 / 1.0$ & 88.0 & 16,013 & 16,296 \\
\hline PDMS (CHDI) & $60.6 / 1.0$ & 89.5 & 16,295 & 16,472 \\
\hline
\end{tabular}

${ }^{1}$ PDMS was reacted with the diisocyanate displayed within the brackets. ${ }^{2}$ Theoretical $\bar{M}_{n}$ was calculated according to $\bar{M}_{n}=($ conversion $/ 100 \%) \cdot \frac{m\left(\mathrm{D}_{4}\right)+m(\text { APTMDS })}{n \text { (APTMDS })} \cdot{ }^{3} \bar{M}_{n}$ was obtained as an average value from four titrations. PDMSs: poly(dimethylsiloxanes); PSU: PDMS-based PUr elastomers; $\mathrm{D}_{4}$ : octamethylcyclotetrasiloxane; APTMDS: 1,3-bis(3-aminopropyl)-tetramethyldisiloxane; $\mathrm{H}_{12}$ MDI: 4,4'-methylenebis(cyclohexyl isocyanate); IPDI: isophorone diisocyanate; MDI: 4,4'-methylenebis(phenyl isocyanate); CHDI: trans-1,4-cyclohexane diisocyanate.

PDMS and APTMDS were dried and degassed under vacuum before use. CHDI was purified by resublimation and distillation under vacuum before use. All other chemicals were used as received.

Human adult calcium high-temperature keratinocytes (HaCaT cells) were obtained from CLS Cell Lines Service GmbH (Eppelheim, Germany). Dulbecco's modified Eagle's medium (DMEM) (high glucose) and fetal bovine serum (FBS) were purchased from Thermo Fisher Scientific Life Technologies GmbH (Darmstadt, Germany). The tetrazolium dye [3-(4,5-dimethylthiazol-2-yl)-5-(3-carboxymethoxyphenyl)-2-(4-sulfophenyl)2H-tetrazolium salt] (MTS) was purchased as a CellTiter96 ${ }^{\circledR}$ Aqueous One Solution cell proliferation assay from Promega $\mathrm{GmbH}$ (Mannheim, Germany).

\subsection{Determination of Molecular Weight}

\subsubsection{Titration}

Number average molecular weights $\left(\bar{M}_{n}\right)$ of the prepared PDMSs (see Table 1) were determined by titration of the amino end groups in THF with $0.1 \mathrm{~N} \mathrm{HCl}$ using bromophenol blue (a detailed procedure is given in [35]). The molecular weight was calculated from an average of four titrations and was used for calculating the reaction stoichiometry of the subsequent PSU syntheses.

\subsubsection{Size-Exclusion Chromatography}

Size-exclusion chromatography (SEC) studies were performed on a 1260 Infinity II GPC-SEC analysis system (Agilent Technologies Deutschland GmbH, Waldbronn, Germany) equipped with 2 PSS SDV columns (Polymer Standards Service) and a refractive index detector. PSU elastomers were dissolved in THF and filtered solutions were measured at $40{ }^{\circ} \mathrm{C}$ and a flow rate of $0.5 \mathrm{~mL} / \mathrm{min}$. Polystyrene standards were used for calibration.

\subsubsection{Determination of Intrinsic Viscosity}

Intrinsic viscosity $([\eta])$ values of the PSU elastomers were determined from PSU solutions in THF using an Ubbelohde viscometer. For each PSU elastomer, measurements were performed on four solutions with different concentrations (c) in the range $0.5-2.0 \mathrm{~g} / \mathrm{dL}$ at $25^{\circ} \mathrm{C}$. Solutions were filtered through a PTFE-membrane filter prior to measurement to exclude any particles. Values for reduced viscosity $\left(\eta_{\text {red }} .=\eta_{\text {spec }} / \mathrm{c}\right)$ were calculated as averages from 10 replicate measurements. Standard deviations of the flow times were 
$\leq 0.7 \%$. Values for $[\eta]$ were obtained as the intercepts in plots of reduced viscosity $\left(\eta_{\text {red. }}\right)$ versus concentration. (See Huggins plots in Figure A2 in Appendix B).

\subsection{Characterization of Molecular Weight}

PSU elastomers were prepared from the diisocyanates $\mathrm{H}_{12} \mathrm{MDI}$, MDI, IPDI, and CHDI according to the prepolymer method. Syntheses were carried out in THF at room temperature or, in the cases of MDI and CHDI, at approximately $0{ }^{\circ} \mathrm{C}$ without a catalyst. The HS contents (see Equation (1)) of the PSU elastomers synthesized from $\mathrm{H}_{12} \mathrm{MDI}$ and IPDI were systematically varied between approximately $1.5 \%$ (PSU without chain extender) and $20 \%$ in steps of $5 \%$. The labeling convention for the PSU elastomers is as follows: PSU diisocyanate-HS content (\%), e.g., $\mathrm{PSU} \mathrm{H}_{12} \mathrm{MDI}-1.6 \%$.

$$
\text { HS content in PSU } \%=\frac{m(\text { diisocyanate })(\mathrm{g})+m(\text { APTMDS })(\mathrm{g})}{m(\text { diisocyanate }+ \text { APTMDS }+ \text { PDMS })(\mathrm{g})}
$$

A typical reaction procedure for the synthesis of a PSU copolymer (PSU $\mathrm{H}_{12} \mathrm{MDI}-$ $1.6 \%$ ) from a PDMS of $\bar{M}_{n}$ : ca. $16,300 \mathrm{~g} \cdot \mathrm{mol}^{-1}$ was as follows: In a $250 \mathrm{~mL}$ four-neck, round-bottom reaction flask equipped with a PTFE centrifugal stirrer, dropping funnel, and nitrogen in- and outlets, $0.65 \mathrm{~g}(2.5 \mathrm{mmol}) \mathrm{H}_{12} \mathrm{MDI}$ was dissolved in $30 \mathrm{~mL}$ THF and added to the reaction flask. Then, $35.0 \mathrm{~g}(2.1 \mathrm{mmol})$ PDMS was dissolved in $100 \mathrm{~mL}$ THF and the PDMS solution was added dropwise to the $\mathrm{H}_{12} \mathrm{MDI}$ solution using the dropping funnel. Approximately $40 \mathrm{~mL}$ of THF, used to rinse the dropping funnel, was also added to the reaction mixture. The reaction progress was monitored using attenuated total reflectionFourier transform infrared (ATR-FTIR) spectroscopy to measure the isocyanate absorption peak at $2265 \mathrm{~cm}^{-1}$. Portions of the PDMS (in steps of $1.5 \mathrm{~g}$ ) dissolved in $10 \mathrm{~mL}$ THF were then added to the reaction mixture. After each addition, an IR spectrum was taken. Polyaddition was assumed complete when the isocyanate absorption peak had completely disappeared from the IR spectrum.

The synthesis of a PSU elastomer containing $20 \%$ HS (PSU $\mathrm{H}_{12}$ MDI-20\%) was performed as follows: First, $5.45 \mathrm{~g}(20.8 \mathrm{mmol}) \mathrm{H}_{12} \mathrm{MDI}$, dissolved in $30 \mathrm{~mL}$ THF, was added to a round-bottom reaction flask. Then, $40.00 \mathrm{~g}(2.5 \mathrm{mmol})$ PDMS was dissolved in $100 \mathrm{~mL}$ THF and added dropwise to the $\mathrm{H}_{12} \mathrm{MDI}$ solution using a dropping funnel. Approximately $40 \mathrm{~mL}$ of THF, used to rinse the dropping funnel, was also added to the reaction mixture. The formation of the prepolymer was monitored via ATR-FTIR spectroscopy. Then, stoichiometric portions of APTMDS (total amount: $4.95 \mathrm{~g} ; 18.3 \mathrm{mmol}$ ), each dissolved in $10 \mathrm{~mL}$ THF, were added dropwise to the prepolymer solution. The reaction progress was followed by monitoring the isocyanate absorption peak. The isocyanate peak disappeared from the IR spectrum after the last portion of APTMDS was added.

The PSU solutions were poured into PTFE-foil-covered glass petri dishes to evaporate the solvent under a fume-hood overnight. Finally, the PSU elastomers were further dried under vacuum at $80^{\circ} \mathrm{C}$ for at least $24 \mathrm{~h}$.

\subsection{Preparation of PSU Elastomer Films for Measurement of Transparency and Mechanical Properties}

Approximately $8 \mathrm{~g}$ of the PSU elastomer were cut into small pieces and dissolved in 200-300 $\mathrm{mL} \mathrm{CHCl}_{3}$ with continuous stirring for at least $24 \mathrm{~h}$ using a magnetic stirrer bar. The homogenous polymer solution was then cast into a glass petri dish and covered with a perforated aluminum foil to slowly evaporate the solvent at room temperature in a well-ventilated location. Finally, the films were dried further in a vacuum chamber at $80^{\circ} \mathrm{C}$ for approximately $12 \mathrm{~h}$ and carefully removed from the glass surface using a small thin spatula. The round edges were cut off and the films with final thicknesses between 0.33 and $0.40 \mathrm{~mm}$ were stored at approximately $23^{\circ} \mathrm{C}$ within a transparent envelope until further characterization. 


\subsection{Measurement of Transparency}

UV-Vis spectroscopy was applied to characterize the transparency of the synthesized PSU films. Film thicknesses ranged between 0.33 and $0.36 \mathrm{~mm}$ (see Table A8 in Appendix F). Measurements of the PSU films were performed on a Lambda1050 UVVis/NIR spectrophotometer (Perkin Elmer LAS GmbH, Rodgau, Germany) between 750 and $200 \mathrm{~nm}$ with a resolution of $2 \mathrm{~nm}$ and against air as the background. The spectra are displayed as the average from three measurements.

\subsection{Measurement of Mechanical Properties}

Tensile tests were performed on PSU films to determine YM, ultimate tensile strength (UTS), elongation at break, and mechanical hysteresis. Tensile and hysteresis measurements were performed on a Zwick model 81,565 tensile testing machine (Zwick GmbH \& Co. KG, Ulm, Germany) using a $100 \mathrm{~N}$ load cell and testXpert II version 3.31 software. Dog-bone shaped specimens according to DIN 53504, type S2 [36] were die cut from these films and stored at $23{ }^{\circ} \mathrm{C}$ for at least $72 \mathrm{~h}$ until measurement. Prior to measurement, all samples were inspected under a cross-polarizer and confirmed free of internal stress. Samples with an original length $\left(\mathrm{L}_{0}\right)$ of $20 \mathrm{~mm}$ were stretched until breaking with a crosshead speed of $25 \mathrm{~mm} / \mathrm{min}$ after reaching a pre-load of $0.1 \mathrm{MPa}$.

The 10-cycle hysteresis behaviors of the PSU elastomers were investigated by stretching the samples to $100 \%$ or $300 \%$ elongation using a crosshead speed of $25 \mathrm{~mm} / \mathrm{min}$. After the samples had reached their desired elongation, they were immediately released at the same crosshead speed. The next cycle was started immediately after the crosshead had returned to the initial starting position.

Hysteresis for each cycle was calculated according to Equation (2):

$$
\text { Hysteresis }(\%)=\frac{\text { area under the loading curve }- \text { area under the recovery curve }}{\text { area under the loading curve }} \cdot 100 \%
$$

Measurements were performed at $23 \pm 2{ }^{\circ} \mathrm{C}$ at a humidity of $55 \pm 5 \%$ and results are reported as mean values of five measurements for tensile tests and three for each hysteresis measurement.

\subsection{Measurement of In Vitro Cytotoxicity}

The in vitro cytotoxicity of the PSU elastomers was measured according to the procedure described in DIN EN ISO 10993-5:2009 [37]. Cell-medium extracts of the elastomers were tested on $\mathrm{HaCaT}$ cells to evaluate whether their low-molecular-weight leachables have an adverse effect on cell proliferation. Cell viability was measured using an MTS cell proliferation assay after culturing the cells at $37 \pm 1{ }^{\circ} \mathrm{C}$ and $5 \% \mathrm{CO}_{2}$ with cell-medium extracts supplemented with $10 \%$ FBS.

$\mathrm{HaCaT}$ cells were cultured according to a protocol described elsewhere [36]. Cells were split at least four times before they were applied in the cytotoxicity assays.

Ethylene-oxide-sterilized samples of the PSUs and a biomedical-grade thermoplastic PU (Carbothane PC-3585A) reference material were weighed into falcon tubes and extracted with DMEM (without FBS) at an extraction ratio of $0.1 \mathrm{~g} / \mathrm{mL}$. Extraction was conducted at $37{ }^{\circ} \mathrm{C}$ and $5 \% \mathrm{CO}_{2}$ atmosphere for $72 \pm 2 \mathrm{~h}$. Comparable volumes of fresh DMEM were pipetted into separate falcon tubes and subjected to the same extraction conditions. The DMEM without PSU was used as blank. After the extraction period, DMEM was immediately supplemented with 10\% FBS. Fresh DMEM supplemented with 10\% FBS was used as negative control. The positive control consisted of FBS-supplemented DMEM containing $1 \%$ sodium dodecyl sulfate.

HaCaT cells were seeded at a concentration of $20 \times 10^{3}$ cells/well in 96-well microplates and incubated for $24 \mathrm{~h}$ at $37^{\circ} \mathrm{C}$ and $5 \% \mathrm{CO}_{2}$ atmosphere. Then, the cell medium was replaced with the extracts and controls, and the cells were incubated at $37^{\circ} \mathrm{C}$ and $5 \% \mathrm{CO}_{2}$ for a further $24 \mathrm{~h}$. Just before the end of the incubation time, a stock solution of MTS was prepared by mixing the MTS dye and DMEM (without FBS) at $20 \mu \mathrm{L}$ MTS + 
$100 \mu \mathrm{L}$ DMEM for each well. After the incubation period, the extracts and controls were removed from the wells and $120 \mu \mathrm{L}$ of the MTS stock solution was pipetted into each well. Additionally, the MTS solution was added to wells without cells for determination of the background.

The microplates were incubated for $4 \mathrm{~h}$ and measured with a microplate reader at a wavelength of $492 \mathrm{~nm}$. In vitro cytotoxicity of each PSU elastomer was assessed by measuring three independent extracts with six replicates for each extract. Thus, 18 measurements in total were performed for each elastomer.

For analysis of cell proliferation, the background value of the MTS solution was subtracted from all absorbance values. All absorbance values of the positive control (no cell viability) were expressed as zero. The values of the negative control (serum-supplemented DMEM) were expressed as $100 \%$ cell proliferation. Absorbance values of the blank and the measured extracts were used to calculate percentage proliferation in comparison to the negative control (100\% proliferation) and the positive control ( $0 \%$ proliferation). PSU extracts were defined as "not-cytotoxic" if the determined cell proliferation was over $81 \%$.

\section{Results and Discussion}

\subsection{Synthesis and Characterization of PDMS-Based PUr Elastomers}

PSU elastomers were synthesized according to a two-step procedure (see Scheme 1). In the first step, the diisocyanate was reacted with the amino-terminated PDMS to yield a PSU prepolymer containing active isocyanate groups at each end of a PDMS chain. In the second step, the remaining isocyanate groups were reacted with the disiloxane-based chain extender APTMDS to build up a segmented polymer containing polar urea HSs and nonpolar PDMS soft segments.

In our previous report, the effect of PDMS molecular weight on the transparency and mechanical properties of urea elastomers was investigated [33]. The results revealed that very soft and transparent urea elastomers were obtained when a PDMS with a molecular weight of approximately $16,000 \mathrm{~g} \cdot \mathrm{mol}^{-1}$ was used. Therefore, the PSU elastomers in this study were prepared from PDMSs with molecular weights ranging between 16,300 and $16,500 \mathrm{~g} \cdot \mathrm{mol}^{-1}$. The HS content and the type of diisocyanate were altered in these PSU elastomers in order to systematically study their effects on the final transparencies and mechanical properties of the elastomers.

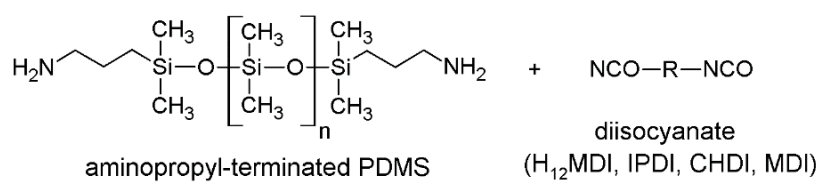
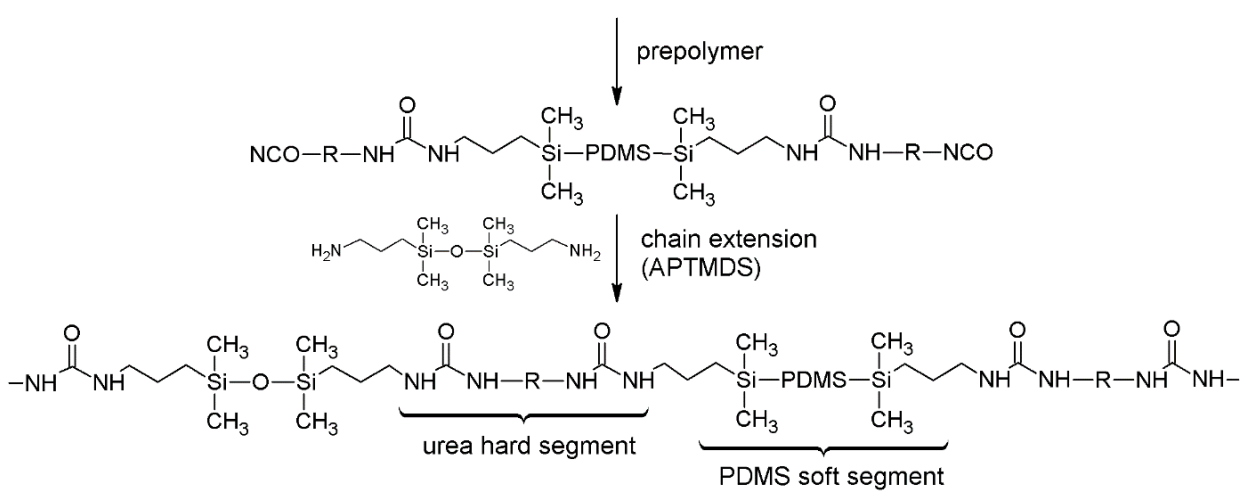

Scheme 1. Synthesis of segmented PDMS-based polyurea elastomers via a two-step synthesis method. A prepolymer comprising active isocyanate groups is formed by reacting excess diisocyanate with amino-terminated PDMS. Segmented PSU elastomers, containing urea hard segments (HSs) and PDMS soft segments, are obtained after chain extension with APTMDS. For PSU copolymers synthesized without chain extender, segmented polymers consisting of alternating sequences of short HSs (exclusively diisocyanate) and long PDMS soft segments $\left(>16,000 \mathrm{~g} \cdot \mathrm{mol}^{-1}\right)$ are formed. 
As the amount of HS in the PSU elastomers increases from $1.5 \%$ to $20 \%$, the molecular weight of the HSs increases linearly from approximately 300 to $4000 \mathrm{~g} \cdot \mathrm{mol}^{-1}$ (see Table 2 and Figure A1 in Appendix A). For the PSU copolymers synthesized without the chain extender, the structures consist of alternating blocks of diisocyanate and long PDMS chains.

Table 2. Molecular weights of the aminopropyl-terminated PDMSs used for synthesis of PSU elastomers in this study.

\begin{tabular}{|c|c|c|c|c|c|c|}
\hline PSU & $\begin{array}{c}\text { Molar Ratio } \\
\text { (PDMS/NCO/CE) }\end{array}$ & $\begin{array}{c}\bar{M}_{n} \\
\operatorname{SEC}\left(\times 10^{3}\right. \\
\left.\mathrm{g} \cdot \mathrm{mol}^{-1}\right)\end{array}$ & $\begin{array}{c}\bar{M}_{w} \\
\mathrm{SEC} \\
\left(\times 10^{3} \mathrm{~g} \cdot \mathrm{mol}^{-1}\right)\end{array}$ & PDI & $\begin{array}{c}\text { Intrinsic } \\
\text { Viscosity }[\eta] \\
\left(\mathrm{dL} \cdot \mathrm{g}^{-1}\right)^{\mathbf{1}}\end{array}$ & $\begin{array}{c}\bar{M}_{n} \\
\mathrm{HS}^{2} \\
\left(\mathrm{~g} \cdot \mathrm{mol}^{-1}\right)\end{array}$ \\
\hline PSU $\mathrm{H}_{12} \mathrm{MDI}-1.6 \%$ * & $1.0 / 1.0 / 0.0$ & 553 & 1185 & 2.14 & 1.05 & 295 \\
\hline PSU $\mathrm{H}_{12} \mathrm{MDI}-5 \%$ & $1.0 / 2.2 / 1.2$ & 547 & 1314 & 2.33 & 0.77 & 892 \\
\hline PSU H $\mathrm{H}_{12} \mathrm{MDI}-10 \%$ & $1.0 / 4.0 / 3.0$ & 441 & 859 & 1.95 & n.a $\#$ & 1848 \\
\hline PSU H ${ }_{12}$ MDI-15\% & $1.0 / 6.1 / 5.1$ & 401 & 832 & 2.07 & n.a $\#$ & 2915 \\
\hline PSU $\mathrm{H}_{12}$ MDI- $20 \%$ & $1.0 / 8.5 / 7.5$ & 314 & 707 & 2.26 & n.a & 4116 \\
\hline PSU IPDI-1.4\% * & $1.0 / 1.0 / 0.0$ & 675 & 1498 & 2.22 & 1.06 & 257 \\
\hline PSU IPDI-5\% & $1.0 / 2.4 / 1.4$ & 487 & 1046 & 2.15 & 0.86 & 892 \\
\hline PSU IPDI-10\% & $1.0 / 4.4 / 3.4$ & 319 & 631 & 1.98 & 0.65 & 1849 \\
\hline PSU IPDI-15\% & $1.0 / 6.7 / 5.7$ & 245 & 506 & 2.06 & 0.48 & 2916 \\
\hline PSU IPDI-20\% & $1.0 / 9.2 / 8.2$ & 116 & 288 & 2.48 & 0.32 & 4126 \\
\hline PSU MDI- $1.5 \%$ * & $1.0 / 1.0 / 0.0$ & 635 & 1853 & 2.92 & 1.16 & 285 \\
\hline PSU CHDI- $1.0 \%$ * & $1.0 / 1.0 / 0.0$ & 403 & 829 & 2.06 & 0.77 & 194 \\
\hline
\end{tabular}

CE: chain extender (APTMDS); PDI: polydispersity index. ${ }^{1}$ Intrinsic viscosity was calculated from Huggins plots (see Figure A2 in Appendix B). Values were taken from the intercepts of the fitting curves. ${ }^{\#}$ No linear relationship was obtained for the PSUs $\mathrm{H}_{12} \mathrm{MDI}-10 \%$ and $\mathrm{H}_{12} \mathrm{MDI}-20 \%{ }^{2}$ The theoretical number average molecular weight of the HSs $\left(\mathrm{g} \cdot \mathrm{mol}^{-1}\right.$ ) was calculated from the reaction stoichiometry. * No chain extender was included in the polymer, and the HS molecular weight resulted from the diisocyanate + terminal urea groups formed by the amino groups of the PDMS. PSU elastomers from $\mathrm{H}_{12} \mathrm{MDI}$ and IPDI were synthesized from a PDMS of $\bar{M}_{n}: 16,338 \mathrm{~g} \cdot \mathrm{mol}^{-1}$. The PSU copolymers from MDI and CHDI were prepared from a PDMS of $\bar{M}_{n}: 16,296 \mathrm{~g} \cdot \mathrm{mol}^{-1}$ and $\bar{M}_{n}: 16,472 \mathrm{~g} \cdot \mathrm{mol}^{-1}$, respectively. (Average values were determined from four titrations).

Table 2 presents the molar ratios of the monomers used for the synthesis of PDMSbased polyurea elastomers, the molecular weights determined by SEC, and $[\eta]$ values. Furthermore, the theoretical number average molecular weights $\left(\bar{M}_{n}\right)$ of the HSs, which were calculated according to the reaction stoichiometry, are also displayed.

All the PSU elastomers exhibit $\bar{M}_{n}$ values higher than $100,000 \mathrm{~g} \cdot \mathrm{mol}^{-1}$ and polydispersity indices (PDIs) between 2.0 and 2.5, except for the copolymer from MDI, which has a PDI close to 3.0. The $\bar{M}_{n}$ values of the PSU elastomers prepared from $\mathrm{H}_{12} \mathrm{MDI}$ and IPDI decrease almost linearly with increasing HS content. Conversely, the PSU copolymers and elastomers comprising an HS content of 5\% exhibit very high number and weight average molecular weights $\left(\bar{M}_{w}\right)$. As expected, the values of $[\eta]$ decrease from 1.16 to $0.32 \mathrm{dL} \cdot \mathrm{g}^{-1}$ when the molecular weights of the PSU elastomers decrease. Interestingly, [ $\eta$ ] seems to be sensitive to the HS content of the PSUs independently of HS structure. In a previous study concerning a series of PSU copolymers synthesized from $\mathrm{H}_{12} \mathrm{MDI}$ and PDMS with molecular weights between approximately 900 and $4000 \mathrm{~g} \cdot \mathrm{mol}^{-1}$, the values for intrinsic viscosity were $0.39,0.45,0.73$, and $0.93 \mathrm{dL} \cdot \mathrm{g}^{-1}$ for $\mathrm{HS}$ contents of $23 \%, 14 \%, 10 \%$, and $6.5 \%$, respectively [38]. These reported values are very close to the values determined here for the PSU IPDI elastomers, even though they are chain extended.

The differences in molecular weights of the PSUs might be attributed to the solubility of the HSs in the solvent used for synthesis of the elastomers, i.e., THF. The PSU copolymers were synthesized without a chain extender and, as a result, the HS molecular weights in these polymers are very low because the HS is exclusively formed by the diisocyanate and the terminal amino groups of the PDMS. Therefore, the local concentration of intermolecular hydrogen bonding between the urea groups is assumed to be low. Furthermore, the polar urea groups are separated from each other by long hydrophobic PDMS chains. Due to the dominantly hydrophobic nature of the PDMS, the copolymers dissolve well in THF. This probably explains the observed moderate viscosity of the polymer solution during synthesis, which may have allowed high-mobility monomers and hence high- 
molecular-weight polymers. However, when the HS content is increased, the HS chain length also increases because a large excess of diisocyanate reacts with the chain extender after formation of the prepolymer. Thus, long sequences of HSs are formed, leading to a high concentration of urea groups. As a result, the ability of the slightly polar solvent to interact with the strongly hydrogen-bonded urea groups is low. During synthesis of these elastomers, the chain extension step is accompanied by a pronounced increase in viscosity, which possibly reduces the mobility of the remaining chain extender molecules.

The observed differences in viscosity can be further explained in terms of the Hildebrand solubility parameters, which are $18.6 \mathrm{MPa}^{1 / 2}$ for THF, $15.6 \mathrm{MPa}^{1 / 2}$ for PDMS, and 45.6 $\mathrm{MPa}^{1 / 2}$ for the urea groups [21,39]. From these solubility parameters, one can deduce that THF preferentially dissolves the PDMS soft segments than the polar urea groups.

The synthesis of soluble and elastic PSU elastomers from the diisocyanates MDI and CHDI with HS contents exceeding 1.5\% failed. Even solvent mixtures of 1:1 tetrahydrofuran/dimethylacetamide and 1:1 tetrahydrofuran/isopropanol were not polar enough to prevent gelation of the PSUs after addition of the chain extender. This behavior probably results from the formation of very strong hydrogen bonds between urea groups in the ordered HSs.

Versteegen et al. synthesized poly(THF)-based PUr copolymers with uniform HSs in order to study the effect of HS size and number of urea groups on the mechanical properties and processabilities of the resultant copolymers [40]. To obtain copolymers with 1-4 urea groups per HS unit, they applied a stepwise synthesis route and, in some cases, employed di-tert-butyl dicarbonate to partially protect the amino groups of the diamine used. Bis(3-aminopropyl)-terminated poly(THF) was either reacted directly with 1,4-diisocyanatobutane or was converted to an NCO-terminated prepolymer by reaction with di-tert-butyl tricarbonate followed by subsequent reaction with 1,4-diaminobutane. The copolymer, which contained two urea groups within the HS unit, exhibited good elastomeric properties and was both meltable and soluble in NMP. However, the copolymers containing three or four urea groups within the HS were neither meltable nor soluble and formed strong physically cross-linked gels. FTIR spectroscopic analysis of the copolymers revealed very strong hydrogen bonding interactions between the monodisperse HSs. Furthermore, they employed either 1,4-diisocyanatobutane or diaminobutane as spacer moieties between the urea groups, both of which are very symmetric due to the even number of methylene groups they contain. For instance, PUs containing 1,4-butane diol rather than 1,5-pentane diol as a chain extender exhibit superior mechanical properties because intermolecular hydrogen bonds are free of strain [41].

In CHDI-based PUr copolymers, the intermolecular hydrogen bonds are planar, which allows very close packing of the HSs in PUs or PUrs. [31] Therefore, this result further confirms the gelling behavior observed during synthesis, particularly that of the CHDIbased PSU elastomers. As is apparent from Figure 2, an HS content of 5\% leads to an average HS repeating unit containing four urea groups. However, increasing the HS contents in the PSU elastomers prepared from the diisocyanates $\mathrm{H}_{12} \mathrm{MDI}$ and IPDI does not lead to such solubility problems during synthesis. $\mathrm{H}_{12} \mathrm{MDI}$ is a mixture of three stereoisomers. The ratio of isomers is approximately $20 \%$ trans-trans-isomer, $50 \%$ cistrans-isomer, and 30\% cis-cis-isomer. IPDI is a mixture of two stereoisomers containing approximately $30 \%$ trans-isomer and $70 \%$ cis-isomer. As $\mathrm{H}_{12} \mathrm{MDI}$ and IPDI are composed of isomeric mixtures containing a low ratio of the symmetric trans-isomer, their HS structures are rather random $[42,43]$. 


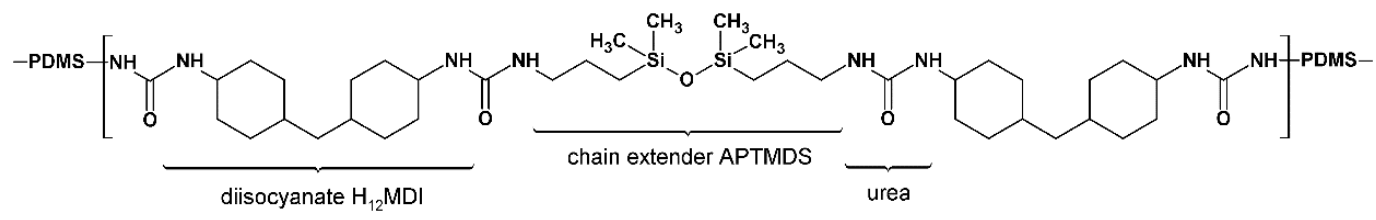

Figure 2. An average HS repeating unit. Example shown is that for the PSU elastomer based on $\mathrm{H}_{12} \mathrm{MDI}$ with an $\mathrm{HS}$ content of 5\%. In this elastomer, the molar ratio of PDMS:NCO:APTMDS is approximately 1:2:1, represented in the structure by the PDMS followed by the HS repeating unit (within brackets) consisting of two diisocyanate molecules and one chain extender molecule. For reasons of simplification, the propyl-chains between the PDMS and urea groups are omitted from this structure.

\subsection{Transparency of PDMS-Based PUr Elastomer Films}

One of the objectives in this study was to study the influence of HS content and isocyanate structure on transparency. This is particularly important for PSU elastomers intended for application as biomaterials in optical applications, e.g., as intraocular lenses or soft contact lenses, where it is important that the materials exhibit a high transmittance within the visible light spectrum. As is apparent from Figures 3 and 4, the PSU elastomers synthesized from $\mathrm{H}_{12} \mathrm{MDI}$ and IPDI are transparent for $\mathrm{HS}$ contents below $10 \%$ and exhibit transmittance values of $90 \%$ and higher at $750 \mathrm{~nm}$. Notably, the spectra of both PSU copolymers reveal high transmittances of over $90 \%$ between $750 \mathrm{~nm}$ and approximately $450 \mathrm{~nm}$, which is similar to that of a 20 diopters intraocular lens [44]. As the HS content increases to $15 \%$ and $20 \%$, the PSU elastomers become increasingly translucent (see photographic images of PSU films in Figure A5 in Appendix D). The transmittances of the PSU films determined at $750 \mathrm{~nm}$ decrease to approximately $85 \%$ and $60 \%$ for HS contents of $15 \%$ and $20 \%$, respectively.
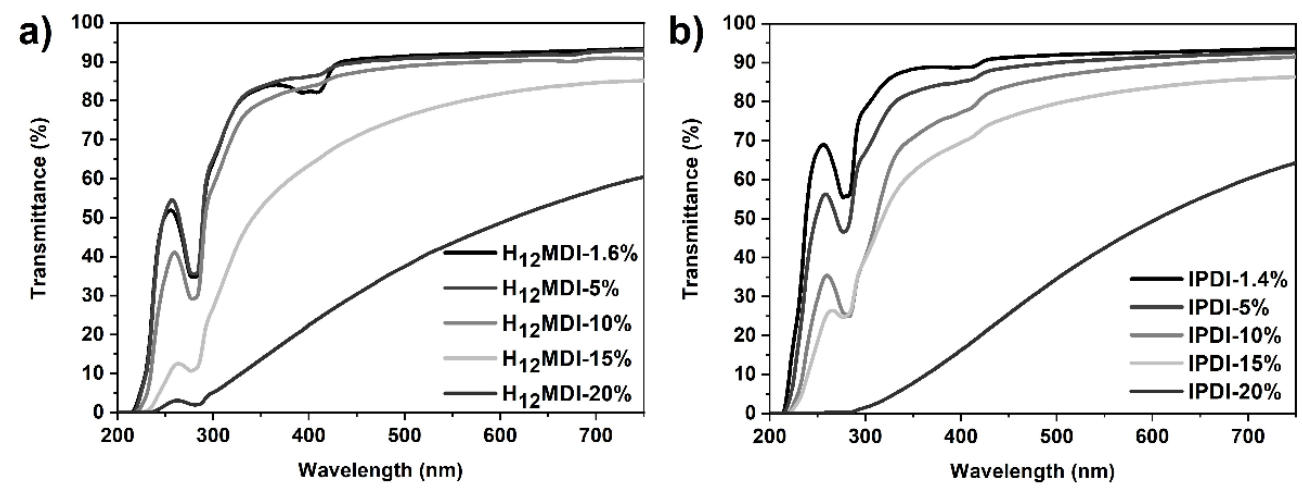

Figure 3. UV-Vis spectra of PSU elastomers synthesized from (a) $\mathrm{H}_{12} \mathrm{MDI}$ and (b) IPDI with HS contents between approximately $1.4 \%$ (copolymers) and 20\%. All PSU elastomers are transparent until an HS content of $10 \%$ and exhibit transmittance values between approximately $91 \%$ and $93 \%$ at $750 \mathrm{~nm}$. The transmittance of the PSU elastomers decreases to approximately $60 \%$ at an HS content of $20 \%$.

Increasing the HS content in the PSU elastomers significantly affects the segmented structure of these polymers. At an HS content of 5\%, the PSU elastomers consist of an alternating sequence of PDMS soft segments separated from each other by one HS repeating unit formed by the diisocyanate and the chain extender APTMDS (see Figure 2). As the HS content doubles from $5 \%$ to $10 \%$, the sequence of PDMS soft segments and urea HSs within these elastomers is significantly altered. As is apparent from the molar ratios displayed in Table 2, approximately $4 \mathrm{~mol}$ of $\mathrm{H}_{12} \mathrm{MDI}$ react with $1 \mathrm{~mol}$ of PDMS in the prepolymer reaction. Thus, the remaining free diisocyanates react with $3 \mathrm{~mol}$ of APTMDS during the chain extension step, leading to a sequence of PDMS soft segments separated by three adjacent repeating HS units. As a result, the HS molecular weight also more than doubles 
from approximately 892 to $1848 \mathrm{~g} \cdot \mathrm{mol}^{-1}$. The HS chain length increases further when the HS content in the PSU elastomers is increased to $15 \%$ and $20 \%$.

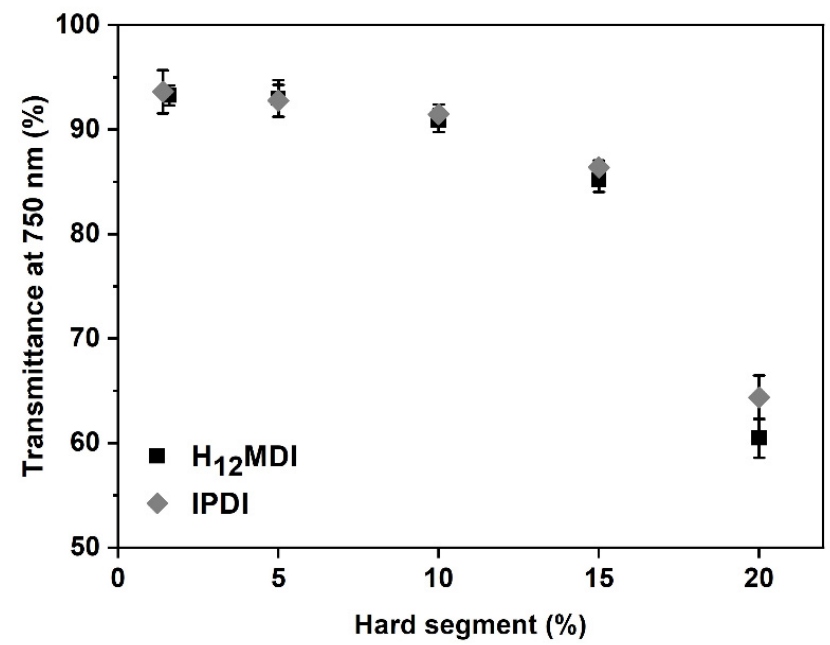

Figure 4. Influence of HS content on transmittance of PSU elastomer films synthesized from $\mathrm{H}_{12} \mathrm{MDI}$ and IPDI. Transmittance at $750 \mathrm{~nm}$ is higher than $90 \%$ in the PSU elastomers with HS contents between $1.4 \%$ and $10 \%$. PSU elastomers with HSs contents of $15 \%$ and $20 \%$ are less translucent and have transmittance values of approximately $85 \%$ and $60 \%$, respectively. The error bars represent the standard deviation from three measurements.

Due to the inherent incompatibility of the nonpolar soft segments with the polar HSs, PUrs, in particular, exhibit a very pronounced microphase separated morphology if no competitive hydrogen bonding between hard and soft segments occurs $[40,45]$. Unlike polyether-based soft segments, PDMS does not participate in intermolecular hydrogen bonding [45]. In these elastomers, urea groups form strong bidentate intermolecular hydrogen bonds, especially within long HS sequences. This leads to domains of wellordered crystalline-like structures that scatter incident light if the aggregates are large enough. The example ATR-FTIR spectra in Figure A4 (see Appendix C) for the PSU $\mathrm{H}_{12} \mathrm{MDI}$ elastomers demonstrate that both $\mathrm{N}-\mathrm{H}$ bonds and carbonyl groups are involved in ordered hydrogen bonding interactions [45].

In a previous study, in which the PDMS soft segment chain length was increased while the HS content of the PSU elastomers was kept constant at 10\%, a comparable opacity was observed in the elastomers synthesized from PDMS with molecular weights of $23,000 \mathrm{~g} \cdot \mathrm{mol}^{-1}$ and higher [32]. The average HS molecular weight in this PSU elastomer was approximately $2600 \mathrm{~g} \cdot \mathrm{mol}^{-1}$, which is close to the HS molecular weight of $2900 \mathrm{~g} \cdot \mathrm{mol}^{-1}$ in the $\mathrm{H}_{12} \mathrm{MDI}$-based elastomer with a $15 \% \mathrm{HS}$ content. As the results of the present and previous studies show, the average HS molecular weight seems to be a critical factor for obtaining either optically clear (if $\bar{M}_{n(H S)}<2200 \mathrm{~g} \cdot \mathrm{mol}^{-1}$ ) or translucent (if $\bar{M}_{n(H S)}>$ $2600 \mathrm{~g} \cdot \mathrm{mol}^{-1}$ ) PSU elastomer films.

Regardless of the diisocyanate used for synthesis, the transmittances of the PSU copolymers within the visible spectrum range (approximately $450-750 \mathrm{~nm}$ ) are equal (see Figure 5). In the MDI-based copolymer, parts of the UV-B range are absorbed by the aromatic rings. When utilizing the PSU elastomers in optical applications such as contact or intraocular lenses, absorption of UV light is favorable because this protects sensitive eye structures and tissues such as the cornea, the crystalline lens, and the retina from the harmful impact of UV radiation. Therefore, the new generation of commercially available intraocular lenses contains chromophores and filters that selectively absorb parts of the blue and UV spectrum [46]. 


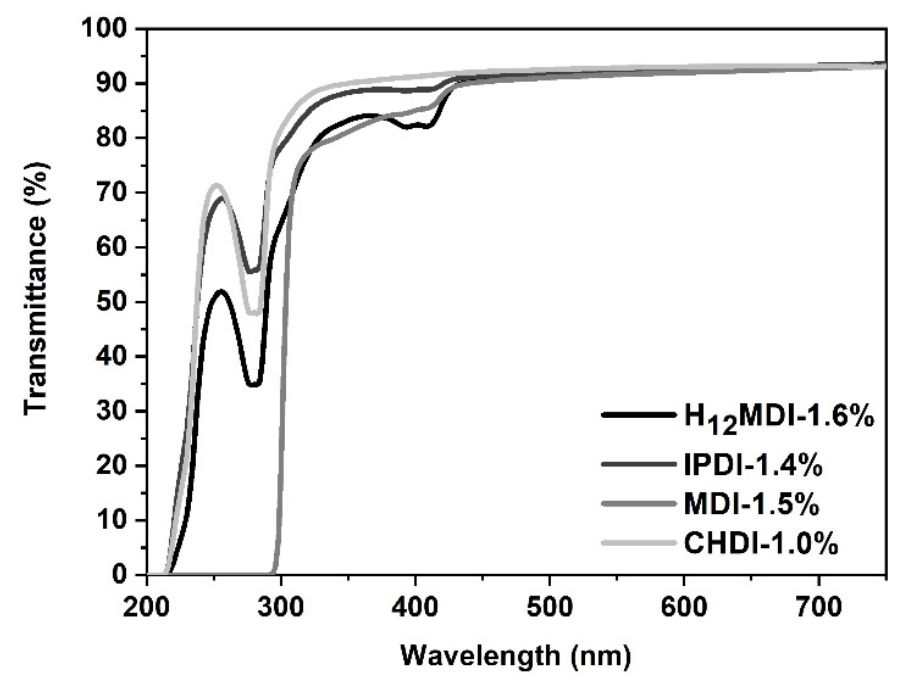

Figure 5. UV-Vis spectra of PSU copolymers synthesized from the aliphatic diisocyanates $\mathrm{H}_{12} \mathrm{MDI}$, IPDI, and CHDI, and from the aromatic diisocyanate MDI without a chain extender. All PSU copolymers exhibit transmittance values of over $90 \%$ between 450 and $750 \mathrm{~nm}$. Transmittance of the PSU copolymer film synthesized from MDI is zero below $300 \mathrm{~nm}$ owing to absorption of UV-B radiation by the aromatic rings.

Interestingly, the MDI-based PSU copolymer does not show any yellowing upon exposure to UV light, which is a typical characteristic in aromatic PUs [47]. It is assumed that the urethane bridge in the aromatic PUs oxidizes to a quinone-imide structure upon exposure to UV light, which itself is a strong chromophore, leading to the yellowing of the polymer [47]. Most likely, the concentration of such structures is too low in the PSU copolymer.

\subsection{Mechanical Properties}

3.3.1. Influence of HS Content on the Mechanical Properties of PDMS-Based PUr Elastomers

As the representative stress-strain curves in Figure 6 show, the mechanical properties, particularly the YM and UTS values, of the PSU elastomers synthesized from $\mathrm{H}_{12} \mathrm{MDI}$ and IPDI are strongly dependent on their HS contents. In both PSU elastomer series, YM increases with the corresponding HS content from approximately 0.52 to $1.34 \mathrm{MPa}$ in the $\mathrm{H}_{12} \mathrm{MDI}$-based elastomers and from 0.27 to $1.28 \mathrm{MPa}$ in the IPDI-based PSU elastomers.

a)

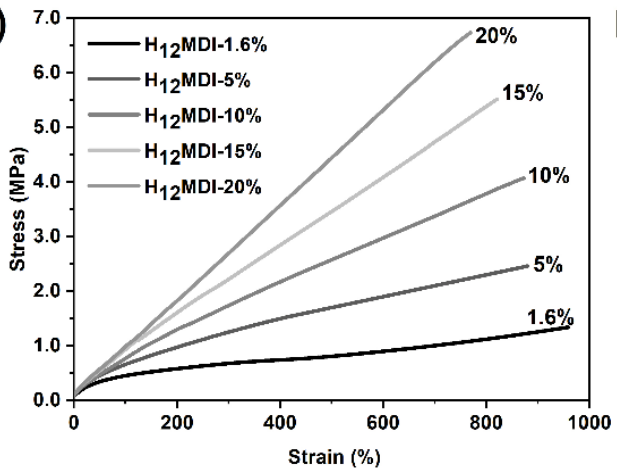

b)

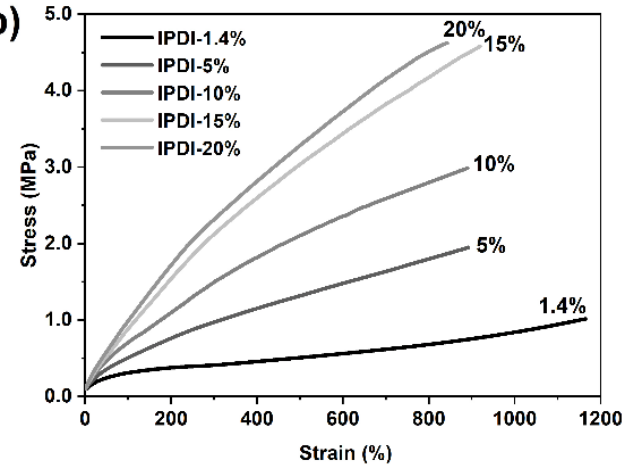

Figure 6. Representative stress-strain curves for PSU elastomers synthesized from (a) $\mathrm{H}_{12} \mathrm{MDI}$ and (b) IPDI with different HS contents. Young's modulus (YM) for the PSUs increase with increasing HS content from approximately $1.4 \%$ to $20 \%$, as is apparent by the steep increase of the slope within the linear ranges of the stress-strain curves. Regardless of their HS contents, all PSU elastomers are highly elastic and exhibited elongation at break values of over $800 \%$. 
In Figure 7, the values of YM are plotted as a function of HS content. When regarding the series of chain-extended PSU elastomers, the values of YM seem to follow a linear trend in the $\mathrm{H}_{12} \mathrm{MDI}$ series and increase in a logarithmical manner in the IPDI series. Prisacariu and Scortanu reported that the YM of aromatic poly(ether urethane)s increases proportionally with HS contents [27]. Conversely, a logarithmic relationship of YM with increasing HS content was reported for copolymers based on PDMS-urea [38] and polyTHFurea [48]. Gaymans et al. synthesized triblock urethane-urea-amide elastomers from PTMO$\mathrm{OH}$ and PTMO prepolymers terminated with MDI and TDI, respectively, and applied the diamine-diamide chain extender $\mathrm{N}$-aminohexyl-N-hexyl-terephthalamide (6T6) [49,50]. In these elastomers, the HS concentration was increased by increasing the PTMO soft segment length. In both series, a linear relationship between the logarithm of the storage modulus and HS content was observed [49,50]. Interestingly, a similar relationship was also reported for polyamides consisting of sebacic acid and hexamethylene diamine. When the amide hydrogens were partially substituted with isobutyl groups, the $100 \%$ modulus showed an approximately logarithmic trend toward lower values with increasing substitution degree [51]. These findings confirm the importance of intermolecular hydrogen bonding for the mechanical properties of amide, urethane, and urea block copolymers.

a)

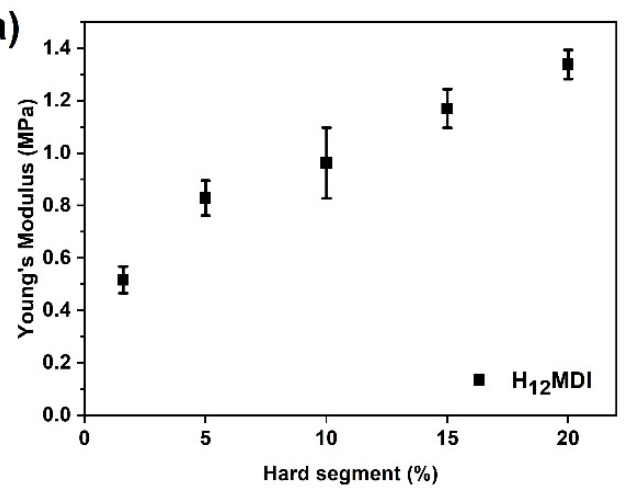

b)

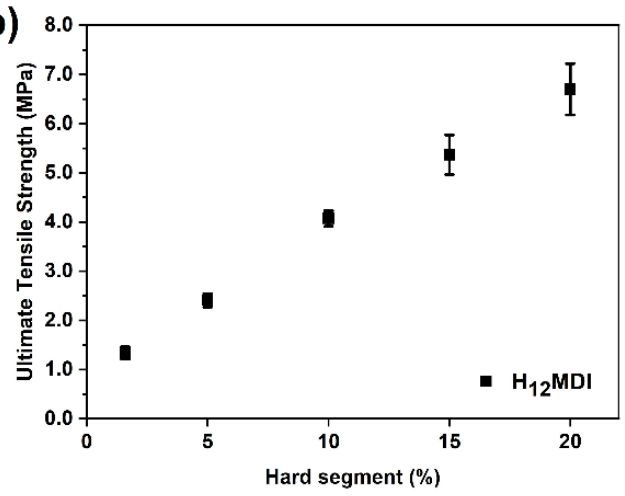

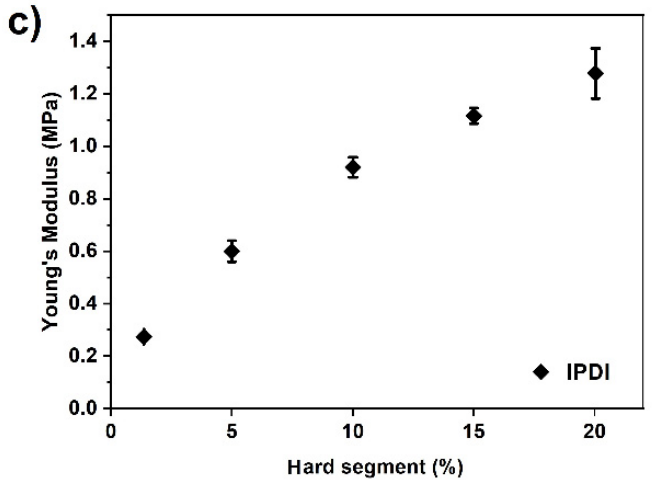

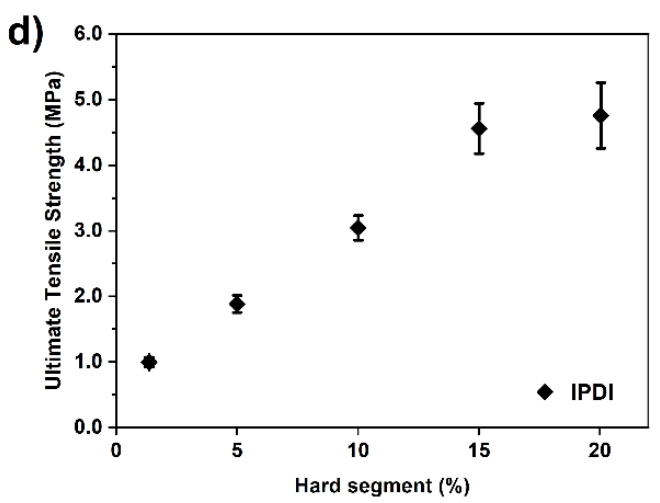

Figure 7. YM and ultimate tensile strength (UTS) values of PSU elastomer films synthesized from $(\mathbf{a}, \mathbf{b}) \mathrm{H}_{12} \mathrm{MDI}$ and $(\mathbf{c}, \mathbf{d})$ IPDI as a function of HS content. The values at approximately $1.6 \%$ and $1.4 \%$ HS content correspond to copolymers that were synthesized without chain extenders. The values between $5 \%$ and $20 \%$ HS content correspond to PSU elastomers that were chain-extended with APTMDS. The YM values of the PSU $\mathrm{H}_{12} \mathrm{MDI}$-elastomers increases approximately linearly from 0.8 to $1.3 \mathrm{MPa}$. In the IPDI series, the values for YM increase logarithmically from approximately 0.6 to $1.3 \mathrm{MPa}$. UTS increases linearly with increasing HS content from 1.3 to $6.7 \mathrm{MPa}\left(\mathrm{H}_{12} \mathrm{MDI}\right)$ and from 1.0 to $4.6 \mathrm{MPa}$ (IPDI). The value at 20\% HS content in the IPDI series deviates slightly from linearity. The error bars represent the standard deviation from five measurements.

At low HS contents (1.4-5\%), the PSU elastomers based on IPDI are softer than those synthesized from $\mathrm{H}_{12} \mathrm{MDI}$, which is most probably an effect of the very asymmetric structure of this diisocyanate. As IPDI is a mixture of two isomers with different symmetries (cis and trans) containing three methyl groups and two chemically different isocyanate 
groups (primary and secondary), the intermolecular distances between the urea groups are not uniform [31]. Therefore, the strength of physical cross-linking within those PSU elastomers, which is important for the mechanical strength of the polymer, is low. However, at HS contents of $10 \%$ and higher, the values of YM in both PSU elastomer series are similar, which might be due to the increasing influence of urea concentration.

Values of UTS are displayed as a function of HS content in Figure $7 \mathrm{~b}$, d. In both series, UTS is directly proportional to HS content, and the values increase from 1.34 to $6.70 \mathrm{MPa}$ $\left(\mathrm{H}_{12} \mathrm{MDI}\right.$ series) and from 1.00 to $4.76 \mathrm{MPa}$ (IPDI series). However, the value for UTS in the IPDI-based elastomer with $20 \%$ HS content deviates from the linear relationship by a factor of approximately 1.0 MPa.

The linear upward trend of tensile strength with increasing HS content (and thus with increasing concentration of urea groups) within the PSU elastomers confirms the findings of other authors. For instance, the Yilgör group conducted systematic studies on the relationship between morphology and mechanical properties in PDMS-based urea copolymers and block copolymers [22,38], poly(propylene oxide) (PPO)-based PUrs [52], and poly(isobutylene)-based urea block copolymers [53]. They reported that, regardless of the type of soft segment applied, a strong linear relationship between the HS content and tensile strength of the polymers is observed. Furthermore, the slopes of the obtained fitting curves did not seem to be greatly influenced by the symmetry of the applied chain extender but were affected by the molecular weight of the soft segment. This observation was explained in terms of a synergistic effect of entanglements arising in soft segments with high molecular weights [22]. A straight line is obtained when plotting stress-at-break against HS content of siloxane-containing poly(urea-oxamide) copolymers [54]. These results reveal that the nature and strength of the hydrogen bonds between the HSs play a major role in determining the extent of microphase separation in segmented PU and PUr elastomers [55]. As the large elongations of the PSU copolymers in Figure 6 and the values for mechanical performance (Figure 7) show, the bidentate urea hydrogen bonds are highly effective for providing sufficient mechanical strength combined with good elastomeric properties, even at very low HS contents.

\subsubsection{Influence of HS Content on Mechanical Hysteresis Behavior in PDMS-Based PUr Elastomers}

Mechanical or tensile hysteresis commonly occurs in thermoplastic PU and PUr elastomers upon repeated loading and unloading cycles due to energy dissipation in the material $[41,56]$. In contrast to conventional rubber-like elastomers, which are covalently cross-linked and hence exhibit very low hysteresis values, segmented PUs and PUrs owe their mechanical stability and elasticity from a network of physical cross-links between urethane and urea groups, respectively, which are embedded in a matrix of amorphous or semi-crystalline soft segments. Upon applied strain, the microstructure of the hard domains is deformed or partially disrupted, leading to a loss of energy due to the conversion of mechanical energy to heat $[42,57]$. During the first loading cycle, the microphase organization within the polymer is markedly changed. As the consecutive relaxation and loading cycles generally proceed at the same speed, the polymer morphology cannot completely regain its initial structure within this short period. Therefore, the consecutive stress values reached at the same applied strain are generally lower leading to differences in hysteresis values between the first and subsequent cycles. This stress softening effect is characteristic for microphase separated polymers [58].

In our previous publication, we reported that hysteresis of PDMS-based PUr elastomers, all with an HS content of $10 \%$, decreases when the molecular weight of the PDMS soft segment, and hence that of the HS, is increased [32]. Therefore, here we studied the effect of HS content on the hysteresis behavior of PSU elastomers prepared from PDMS with a constant molecular weight. In this study, a PDMS with a molecular weight of approximately $16,300 \mathrm{~g} \cdot \mathrm{mol}^{-1}$ was selected because the PSU elastomers we prepared previously from PDMSs with molecular weights between 15,000 and $18,000 \mathrm{~g} \cdot \mathrm{mol}^{-1}$ were transparent and had sufficient mechanical stability and low hysteresis values. When utilizing PDMS- 
based PUr elastomers in biomedical applications, such as wound dressings, vascular grafts, and intraocular lenses, it is crucial for their long-term performance that the materials do not undergo a large irreversible mechanical deformation upon repeated application of strain.

The 10-cycle hysteresis behavior was studied at two strains, $100 \%$ and $300 \%$, because a previous study performed on a poly(ether urethane) revealed that the extent of hysteresis is not only sensitive to morphological changes but also increases with strain [57].

The values of tensile hysteresis, determined for the PSU elastomers based on $\mathrm{H}_{12} \mathrm{MDI}$, are displayed in Figure 8 and Table 3. As expected, the hysteresis values in the first cycles are distinctly higher than those in the subsequent cycles, irrespective of applied strain. After the strain induced morphological reorganization of the HSs during the first stretch, the deformed HS domains remain largely unaffected by the applied strain in the following loading cycles, which is why the values decrease only very slightly or remain constant. Interestingly, the $100 \%$-hysteresis values (1st cycle) of the PSU copolymer and the PSU elastomer containing $20 \% \mathrm{HS}$ are quite similar. This leads to the assumption that hysteresis behavior is dependent on both the concentration of urea groups in the HS and the related hard domain morphology, i.e., randomly dispersed or interconnected.

a)

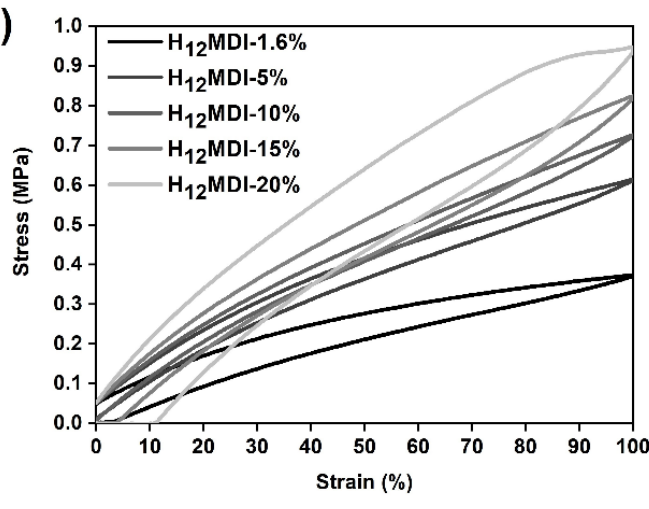

c)

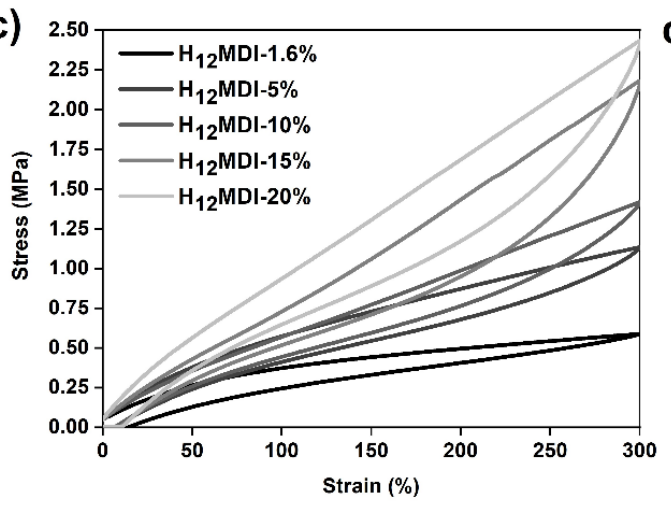

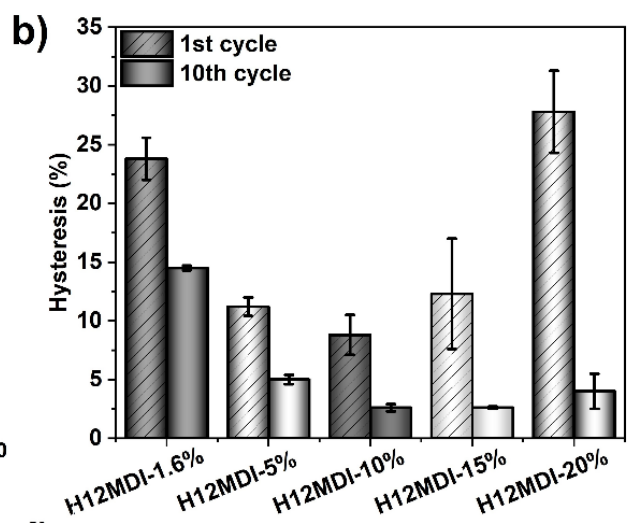

d)

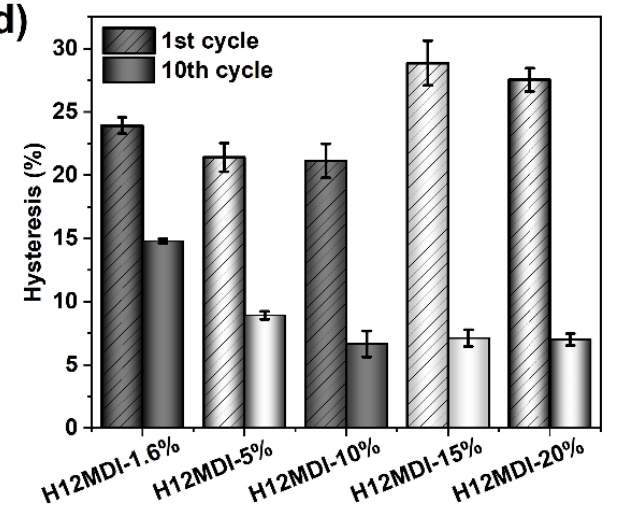

Figure 8. Results of tensile hysteresis measurements on PSU elastomers synthesized from $\mathrm{H}_{12} \mathrm{MDI}$ with HS contents between $1.6 \%$ (copolymer) and $20 \%$. (a) 100\%-hysteresis curves (1st cycle) and corresponding mean hysteresis values (b) of the 1st and 10th cycles. (c) 300\%-hysteresis curves (1st cycle) and corresponding mean hysteresis values of the elastomers (d) at the 1st and 10th cycles. The error bars represent the standard deviation of three repeated measurements. Hysteresis at $100 \%$ strain in the 1 st cycle decreases from approximately $24 \%$ (1.6\% HS content) to $9 \%$ (10\% HS content) and increases again to approximately $28 \%$ at $20 \% \mathrm{HS}$ content. Hysteresis in the 10th cycle decreases from approximately $15 \%(1.6 \% \mathrm{HS}$ content) to $3 \%$ (10\% HS content) and remains constant with increasing HS content. A similar but less pronounced trend for the hysteresis values appears when the PSU elastomers are repeatedly stretched to $300 \%$. 
Table 3. Influence of HS content on tensile hysteresis for PSU elastomers synthesized from $\mathrm{H}_{12} \mathrm{MDI}$ with HSs ranging from $1.6 \%$ (copolymer) to $20 \%$. The values are given for the first three cycles and the last (10th) cycle at 100\% and 300\% strain, respectively. The given values are the mean values from three repeated measurements and were rounded to integers.

\begin{tabular}{lcccc}
\hline \multicolumn{1}{c}{ PSU (Elongation)/Cycle } & $\mathbf{1}$ & $\mathbf{2}$ & $\mathbf{3}$ & $\mathbf{1 0}$ \\
\hline $\mathrm{H}_{12} \mathrm{MDI}-1.6 \%(100 \%)$ & 24 & 16 & 16 & 15 \\
$\mathrm{H}_{12} \mathrm{MDI}-1.6 \%(300 \%)$ & 24 & 17 & 16 & 15 \\
$\mathrm{H}_{12} \mathrm{MDI}-5 \%(100 \%)$ & 11 & 7 & 6 & 5 \\
$\mathrm{H}_{12} \mathrm{MDI}-5 \%(300 \%)$ & 21 & 12 & 11 & 9 \\
$\mathrm{H}_{12} \mathrm{MDI}-10 \%(100 \%)$ & 9 & 4 & 3 & 3 \\
$\mathrm{H}_{12} \mathrm{MDI}-10 \%(300 \%)$ & 21 & 10 & 8 & 7 \\
$\mathrm{H}_{12} \mathrm{MDI}-15 \%(100 \%)$ & 12 & 4 & 3 & 3 \\
$\mathrm{H}_{12} \mathrm{MDI}-15 \%(300 \%)$ & 29 & 11 & 9 & 7 \\
$\mathrm{H}_{12} \mathrm{MDI}-20 \%(100 \%)$ & 28 & 5 & 5 & 4 \\
$\mathrm{H}_{12} \mathrm{MDI}-20 \%(300 \%)$ & 28 & 10 & 9 & 7 \\
\hline
\end{tabular}

When regarding the $100 \%$-hysteresis values of the first cycles in this PSU series, it appears that hysteresis is determined by two different trends. At low HS contents (between $1.6 \%$ and $10 \%)$, hysteresis decreases from approximately $24 \%\left(\mathrm{H}_{12} \mathrm{MDI}\right.$ copolymer) to a minimum of $9 \%$ (PSU $\mathrm{H}_{12} \mathrm{MDI}-10 \%$ ). At higher HS contents (15\% and $20 \%$ ) the hysteresis values increase from $12 \%$ to $28 \%$, respectively. A similar trend is obvious for the values after the 10th cycle. However, for HS contents of $10 \%$ and higher, the hysteresis values remain constant.

Repeated cycling of the PSU elastomers at 300\% strain presents a comparable, though less pronounced, trend of the hysteresis values in the first cycles. Hysteresis slightly decreases from $24 \%$ in the $\mathrm{H}_{12} \mathrm{MDI}$ copolymer to $21 \%$ in the PSU elastomers containing $5 \%$ and $10 \%$ HS. At HS contents higher than 10\%, hysteresis increases again to approximately $29 \%$. After the 10th cycle, hysteresis decreases from $15 \%\left(\mathrm{H}_{12} \mathrm{MDI}\right.$ copolymer) to $7 \%$ (PSU $\mathrm{H}_{12} \mathrm{MDI}-10 \%$ ) and remains constant at higher HS contents.

Looking closer at the hysteresis values for the chain-extended PSU elastomers, it seems that changes in HS content between $10 \%$ and $20 \%$ do not significantly influence hysteresis behavior in the subsequent cycles as the values in each strain series $(100 \%$ and $300 \%$ ) are approximately equal. In other words, once the hard domains are deformed or partially disrupted by the breaking of inter-urea hydrogen bonds, which is most probably the case at high strains, the higher number of urea groups within the HSs obviously does not contribute to mechanical stabilization of the polymer morphology. Furthermore, when considering the unloading cycles of the PSUs with HS contents of $15 \%$ and $20 \%$, it becomes apparent that the curves reach a stress value of zero before the elastomer is completely released from strain. This behavior, often referred to as "instantaneous set," results from energy loss during plastic deformation of the sample [56]. This observation confirms that, particularly at $300 \%$ strain, some of the hydrogen bonds are disrupted because all the PSU elastomers exhibit a similar amount of set after the first and 10th cycle (see hysteresis curves of the 10th cycle in Figure A7 in Appendix E).

In contrast to the chain-extended PSU elastomers, where hysteresis is also dependent on the level of deformation, the hysteresis values in the $\mathrm{H}_{12} \mathrm{MDI}$ copolymer are equal irrespective of whether the copolymer is stretched to $100 \%$ or $300 \%$. Similar behavior is also exhibited by the PSU copolymer synthesized from IPDI, albeit with most hysteresis values being slightly lower at 300\% strain, as the graphs in Figure 9 and the values in Table 4 show. This indicates that the hydrogen bonds between single urea groups that are rather randomly dispersed within the PDMS matrix are weak and as a result are easily disrupted at a low strain level. Moreover, both copolymers displayed a set in the first cycle of the $100 \%$ hysteresis curves, while the PSU elastomers containing four and eight urea groups within one HS repeating unit did not exhibit this behavior. 
a)

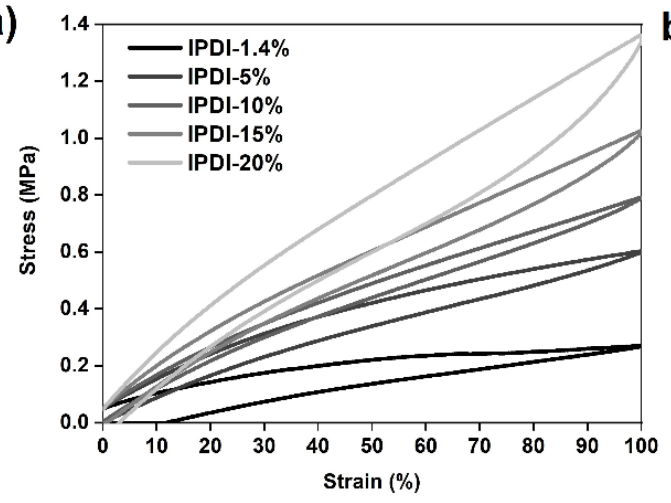

c)

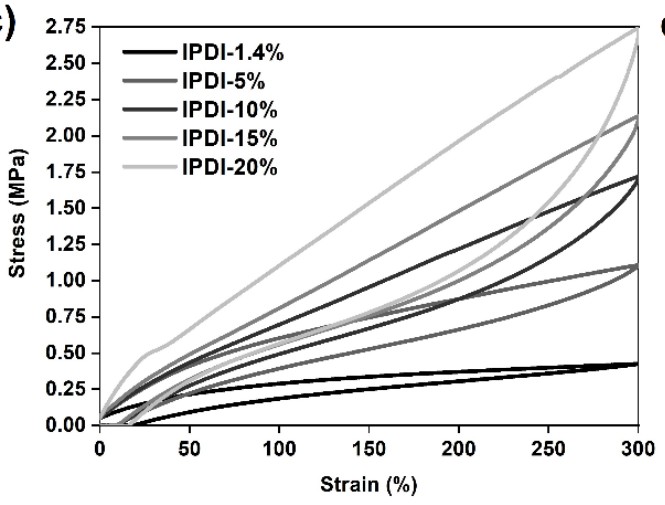

b)

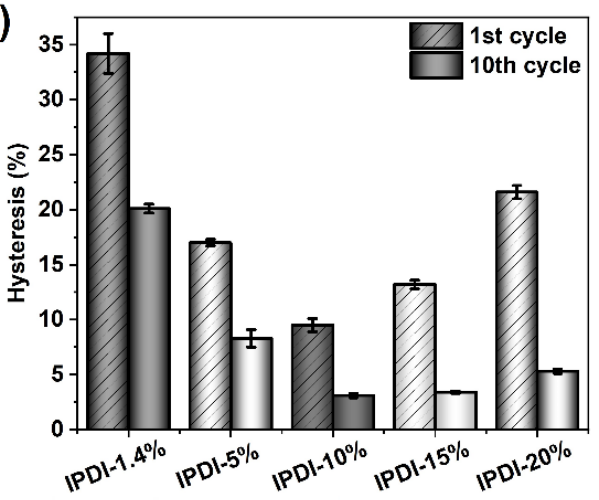

d)

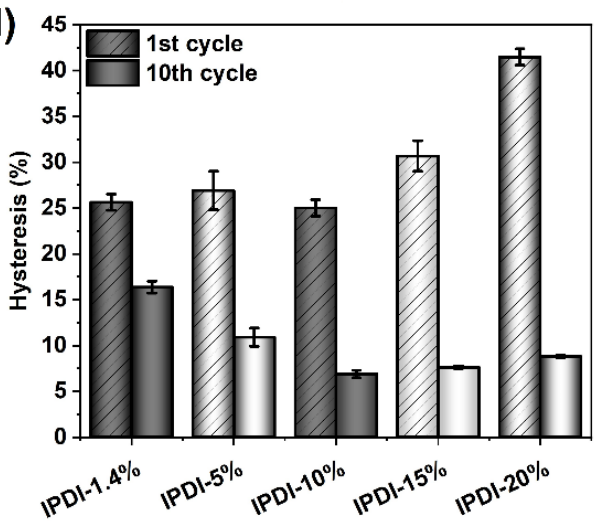

Figure 9. Results of tensile hysteresis measurements on PSU elastomers synthesized from IPDI with HS contents between $1.4 \%$ (copolymer) and $20 \%$. (a) 100\%-hysteresis curves (1st cycle) and corresponding mean hysteresis values (b) of the 1st and 10th cycles. (c) 300\%-hysteresis curves (1st cycle) and corresponding mean hysteresis values of the elastomers (d) at the 1st and 10th cycles. The error bars represent the standard deviation of three repeated measurements. Hysteresis at $100 \%$ strain in the 1 st cycle decreases from approximately $34 \%$ (1.4\% HS content) to 10\% (10\% HS content) and increases again to approximately $22 \%$ at $20 \% \mathrm{HS}$ content. Hysteresis in the 10 th cycle decreases from $20 \%(1.4 \%$ HS content) to $3 \%$ (10\% HS content) and slightly increases again to $5 \%$ at an HS content of $20 \%$. At $300 \%$ strain, the lowest 1st-cycle-hysteresis value is measured in the PSU elastomer containing $10 \%$ HS. The highest hysteresis is over $41 \%$, as observed for the elastomer with an HS content of $20 \%$.

Table 4. Influence of HS content on tensile hysteresis for PSU elastomers synthesized from IPDI with HS contents ranging from $1.4 \%$ (copolymer) to $20 \%$. The values are given for the first three cycles and the last (10th) cycle at $100 \%$ and $300 \%$ strain. The given values are the mean values from three repeated measurements and are rounded to integers.

\begin{tabular}{lcccc}
\hline \multicolumn{1}{c}{ PSU (Elongation)/Cycle } & $\mathbf{1}$ & $\mathbf{2}$ & $\mathbf{3}$ & $\mathbf{1 0}$ \\
\hline IPDI-1.4\% (100\%) & 34 & 21 & 20 & 20 \\
IPDI-1.4\% (300\%) & 26 & 18 & 17 & 16 \\
IPDI-5\% (100\%) & 17 & 10 & 9 & 8 \\
IPDI-5\% (300\%) & 27 & 15 & 13 & 11 \\
IPDI-10\% (100\%) & 10 & 5 & 4 & 3 \\
IPDI-10\% (300\%) & 25 & 10 & 9 & 7 \\
IPDI-15\% (100\%) & 13 & 5 & 4 & 3 \\
IPDI-15\% (300\%) & 31 & 11 & 10 & 8 \\
IPDI-20\% (100\%) & 22 & 8 & 7 & 5 \\
IPDI-20\% (300\%) & 42 & 13 & 12 & 9 \\
\hline
\end{tabular}

The effect of HS content on the 10-cycle hysteresis behavior was additionally studied in an analogous series of PSU elastomers containing the diisocyanate IPDI. As the values 
in Figure 9 and Table 4 show, a similar trend for hysteresis behavior to that of the $\mathrm{H}_{12} \mathrm{MDI}$ series is observed. 100\%-hysteresis in the 1st cycle decreases from $34 \%$ (IPDI copolymer) to a minimum of $10 \%$ in the PSU elastomer containing $10 \%$ HS. Upon further increase of the HS content from $15 \%$ to $20 \%$, hysteresis increases from $13 \%$ to approximately $22 \%$, respectively. After the 10th cycle, hysteresis decreases dramatically from 20\% (IPDI copolymer) to 3\% in the IPDI elastomers containing $10 \%$ and $15 \%$ HS and slightly increases again to $5 \%$ in the PSU IPDI-20\% elastomer.

As already shown for the PSU $\mathrm{H}_{12} \mathrm{MDI}$ series, the trend in hysteresis at $300 \%$ strain is also less pronounced in the PSU IPDI series. Hysteresis in the IPDI-based elastomers, particularly at low HS contents, is higher than in the corresponding elastomers containing $\mathrm{H}_{12} \mathrm{MDI}$. This may be attributed to the effect of less-ordered hydrogen bonds within small particulate hard domains. However, at HS contents of $10 \%$ and higher, this supposed symmetry effect may not be valid as the values of hysteresis in the first and last cycle are approximately equal in both the $\mathrm{H}_{12} \mathrm{MDI}$ and IPDI series. Overall, the highest obtained hysteresis is $41.5 \%$, as observed in the PSU IPDI-20\% elastomer.

The trend of the first-cycle values obtained for both PSU series between $1.6 \%(1.4 \%)$ and $10 \%$ HS content is contrary to the hysteresis results reported by Yilgör et al., who synthesized PDMS-based PUr elastomers from $\mathrm{H}_{12} \mathrm{MDI}$ and the chain extenders 1,2 ethylenediamine and 2-methyl-1,5-diaminopentane [56]. His group studied the effect of PDMS molecular weight, chain extender structure, and HS content on the $200 \%-$ and $300 \%$ hysteresis behavior of PSU elastomers. For a series of elastomers synthesized from PDMS with a molecular weight of $31,500 \mathrm{~g} \cdot \mathrm{mol}^{-1}$, they reported that first-cycle hysteresis values (300\% strain) increased linearly from $12 \%$ to $26 \%$ when the HS content increased slightly from $2.8 \%$ to $4.7 \%$. However, in another series of PSUs synthesized from a PDMS of approximately $11,000 \mathrm{~g} \cdot \mathrm{mol}^{-1}$ with HS contents between $7.8 \%$ and $11.4 \%$, which corresponds to an equal increase of HS molecular weight, the $200 \%$ hysteresis values did not increase significantly [21,56].

This clearly indicates that the HS content and its molecular weight in the PSU elastomer do not determine the hysteresis behavior exclusively. Furthermore, other factors such as soft segment molecular weight and nature of the HSs (crystallizing or not crystallizing) have a major influence on microphase morphology and hence on mechanical behavior [59]. In particular, 1,2-ethylenediamine is a very short and symmetric chain extender, thus promoting the formation of ordered and crystalline HSs. Conversely, the disiloxane-based chain extender applied in this study is very flexible owing to the large $\mathrm{Si}-\mathrm{O}-\mathrm{Si}$ bond angle $\left(145-150^{\circ}\right.$ in hexamethyldisiloxane) and rather mimics the properties of the soft segment [60]. For instance, Adhikari et al. reported that incorporation of $40 \mathrm{~mol} \%$ of the disiloxane-based chain extenders 1,3-bis(4-hydroxybutyl) and (3-hydroxypropyl)tetramethyldisiloxane along with 1,4-butanediol increased the solubilities of the HSs in the PDMS/PHMO soft phases [61,62]. Yildirim et al. simulated the morphology of segmented aliphatic silicone-urea copolymers with varying HS contents using DPD calculations [63]. Models for PSU copolymers revealed a microphase separated morphology even at the very low HS content of $1.7 \%$. When the HS content was increased by decreasing the PDMS molecular weight, the morphology changed from spherical randomly dispersed hard domains to large ribbon-like crystalline hard domains that became interpenetrating, particularly at high urea HS contents [63].

Therefore, in the present study we assumed that the PSU morphology consists of small randomly distributed hard domains surrounded by a continuous phase of PDMS at low HS contents between $1.4 \%$ and $5 \%$. Even though the concentration of urea groups within the HSs is low, they clearly mechanically stabilize the soft segment chains by intermolecular hydrogen bonds, which act as a reinforcing filler. As a result, the hysteresis in the PSU elastomers is decreased. At an HS content of $10 \%$, the hard domains are larger but presumably still discrete. However, when the HS content is increased further to $15 \%$ and $20 \%$, the high concentration of urea groups within the HS repeating units leads to large thread-like hard domains with some crystallinity. This may also explain why the 
transparency of these PSU films decreases with increasing HS content. Such large hard domains are expected to be less flexible under mechanical stress, leading to disruption of the hydrogen bonds, even at low strains of $100 \%$.

Abouzahr et al. investigated the structure property relationship for poly(ether urethane)s containing HSs formed by MDI and 1,4-butanediol with contents between $15 \%$ and $45 \%$ [64]. Their SAXS results revealed that the morphologies of the polymers changed with increasing HS content. At an HS content of $15 \%$, some of the hard domains were solubilized in the soft phase. Isolated hard domains were found in the polymer with a $25 \% \mathrm{HS}$ content. This PU exhibited good elastomeric properties and low hysteresis. At high HS contents (35-45\%), the hard domains became interlocking and, as a result, hysteresis in those PUs was very high. This observation was explained in terms of an irreversible restructuring of the hard domain morphology. Furthermore, it was observed that disruption of the hard domains began at low elongations.

Wang et al. made similar observations for poly(ether urethane urea)s with HS contents between $36 \%$ and $46 \%$ containing 1,2-ethylenediamine [65]. They also observed high initial hysteresis at low strains in those polymers. In addition, they reported that the concentration of hydrogen bonded urea groups decreases after mechanical deformation.

3.3.3. Influence of Diisocyanate Structure on the Mechanical Properties and Hysteresis Behavior of PSU Copolymers

The mechanical properties and hysteresis behavior of a series of PSU copolymers based on diisocyanates with different symmetries synthesized without a chain extender was investigated.

The ability of the urea groups to form strong intermolecular hydrogen bonds largely depends on the order of aligned HSs, which in turn determines the density of packed chains. The degree of microphase separation in PUs and PUrs generally increases if they contain a high concentration of hydrogen bonded urethane or urea groups and if they exhibit a distinct domain structure comprising crystalline hard and amorphous soft segments. Therefore, we selected the highly symmetric CHDI and compared the results of its tensile and hysteresis measurements with those for PSU copolymers based on the asymmetric IPDI and the diisocyanates $\mathrm{H}_{12} \mathrm{MDI}$ and MDI.

The representative stress-strain curves of the copolymers are displayed in Figure 10. As is apparent from the slopes of the stress-strain curves and the mechanical properties shown in Figure 11, the copolymers based on $\mathrm{H}_{12} \mathrm{MDI}$ and MDI both exhibit $\mathrm{YM}$ values of ca. 0.5 MPa. Not surprisingly, the YM of the CHDI-based copolymer is highest in this series and almost twice those of the copolymers based on $\mathrm{H}_{12} \mathrm{MDI}$ and MDI. Conversely, the IPDI-based copolymer exhibits a YM of approximately $0.3 \mathrm{MPa}$, the lowest in this copolymer series.

An approximately similar trend is observed for the UTSs. The PSU copolymers based on $\mathrm{CHDI}, \mathrm{H}_{12} \mathrm{MDI}$, and MDI exhibit similar UTS values, i.e., between 1.2 and $1.4 \mathrm{MPa}$. The lowest UTS of 1.0 MPa is observed for the IPDI-based copolymer. One would expect that the CHDI-based copolymer would also have the highest UTS because its HSs are presumably highly ordered. However, as this elastomer is stiffer than all the others in this series, its elasticity as reflected by its elongation at break is significantly lower (Figure 12). Conversely, the elastomers based on MDI and IPDI are highly elastic and present elongation values of $1050 \%$ and $1080 \%$. 


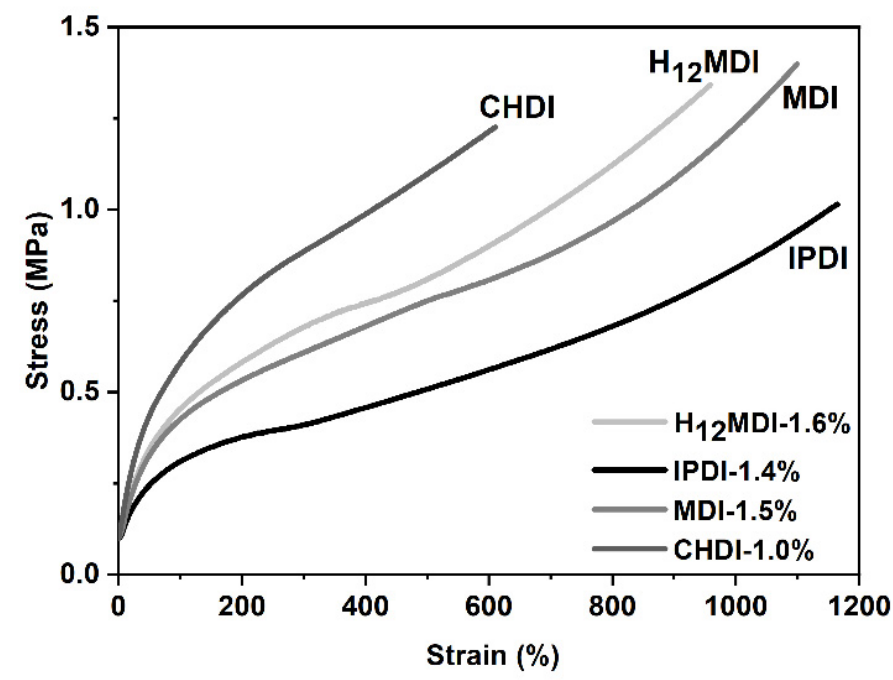

Figure 10. Representative stress-strain curves of PSU copolymers synthesized from the aliphatic diisocyanates $\mathrm{H}_{12} \mathrm{MDI}$, IPDI, and $\mathrm{CHDI}$ and the aromatic diisocyanate MDI without chain extenders. The YM is highest for the CHDI-based copolymer, as is apparent from the steep increase of stress within the linear range of the stress-strain curves. The stress-strain curves of the copolymers based on $\mathrm{H}_{12} \mathrm{MDI}$ and MDI present approximately equal slopes and exhibit elongations of more than $800 \%$. The lowest YM value is presented by the curve of the IPDI-based copolymer.

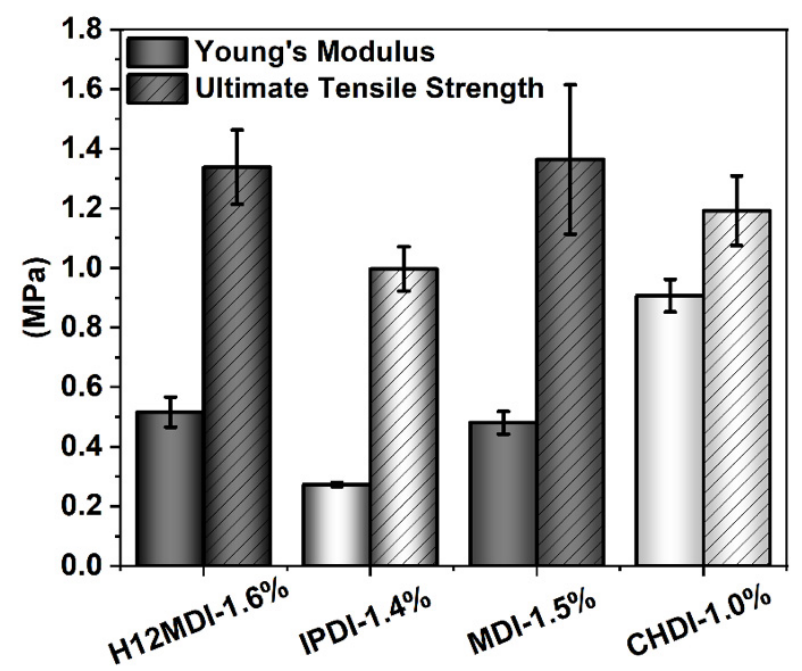

Figure 11. YM (left columns) and UTS (striped-columns) values for PSU copolymers synthesized from the aliphatic diisocyanates $\mathrm{H}_{12} \mathrm{MDI}$, IPDI, and $\mathrm{CHDI}$, and from the aromatic diisocyanate MDI without chain extenders. The error bars represent the standard deviation from five repeated measurements. YM is lowest for the unsymmetric diisocyanate IPDI and highest for the very symmetric diisocyanate CHDI. The YM and UTS values are approximately equal for the PSU copolymers synthesized from $\mathrm{H}_{12} \mathrm{MDI}$ and $\mathrm{MDI}$.

In the previous subsection, in which hysteresis behavior was investigated as function of HS content, it became obvious that a slight increase in HS content led to a mechanical stabilization of the physically cross-linked polymer network. As a result, the hysteresis values decreased and reached a minimum at 10\% HS content. Conversely, higher urea concentrations made the HSs increasingly prone to deformation or disruption of the intermolecular hydrogen bonds upon applied strain. As the copolymers were solely synthesized from the diisocyanate and the amino-terminated PDMS, the HS contents and their molecular weights were low. Hence, the extent of hysteresis was expected to be 
dependent only on the degree of ordered HSs, which is in turn governed by the symmetry of the diisocyanate.

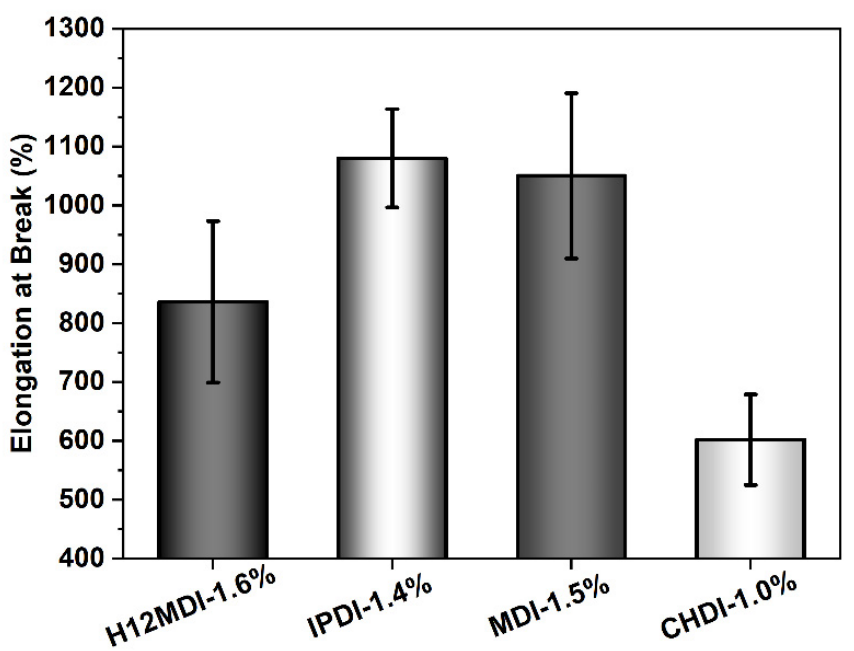

Figure 12. Elongation at break values for PSU copolymers synthesized from the aliphatic diisocyanates $\mathrm{H}_{12} \mathrm{MDI}$, IPDI, and $\mathrm{CHDI}$, and from the aromatic diisocyanate MDI without chain extenders. The error bars represent the standard deviation from five repeated measurements. Except for the CHDI-copolymer, which exhibits an elongation of approximately $600 \%$, all the PSU copolymers are highly elastic with elongations between $800 \%$ and $1100 \%$.

Supposing that the HS order is highest in the CHDI-based elastomer and lowest in the IPDI-based elastomer, one would expect to observe the lowest hysteresis for the CHDI-elastomer because hydrogen bonds in those HSs are free of strain and therefore very strong. This assumption seems to be true in the range of low strains, where indeed the mean hysteresis in the first cycle is 19\% for the CHDI-based elastomer and $34 \%$ for the elastomer synthesized from IPDI, as the bars in Figure 13c show. A clear difference in hysteresis behavior between the CHDI- and IPDI-based elastomers is apparent after the 10th cycle. As the values in Table 5 show, hysteresis for the elastomer containing CHDI decreases to $9 \%$ at $100 \%$ strain and to $13 \%$ at $300 \%$ strain. However, hysteresis in the IPDI-based elastomer remains high at $16 \%$ and $20 \%$.

Table 5. Influence of HS content on tensile hysteresis of PSU copolymers synthesized from the diisocyanates $\mathrm{H}_{12} \mathrm{MDI}$, IPDI, MDI, and CHDI. The values are given for the first three cycles and the last (10th) cycle at $100 \%$ and $300 \%$ strain. The given values are the mean values from three repeated measurements and are rounded to integers.

\begin{tabular}{lcccc}
\hline \multicolumn{1}{c}{ PSU (Elongation)/Cycle } & $\mathbf{1}$ & $\mathbf{2}$ & $\mathbf{3}$ & $\mathbf{1 0}$ \\
\hline $\mathrm{H}_{12} \mathrm{MDI}-1.6 \%(100 \%)$ & 24 & 16 & 16 & 15 \\
$\mathrm{H}_{12} \mathrm{MDI}-1.6 \%(300 \%)$ & 24 & 17 & 16 & 15 \\
$\mathrm{IPDI}-1.4 \%(100 \%)$ & 34 & 21 & 20 & 20 \\
$\mathrm{IPDI}-1.4 \%(300 \%)$ & 26 & 18 & 17 & 16 \\
$\mathrm{MDI}-1.5 \%(100 \%)$ & 23 & 16 & 15 & 14 \\
$\mathrm{MDI}-1.5 \%(300 \%)$ & 32 & 22 & 20 & 18 \\
$\mathrm{CHDI}-1.0 \%(100 \%)$ & 19 & 12 & 11 & 9 \\
$\mathrm{CHDI}-1.0 \%(300 \%)$ & 37 & 21 & 18 & 13 \\
\hline
\end{tabular}


a)

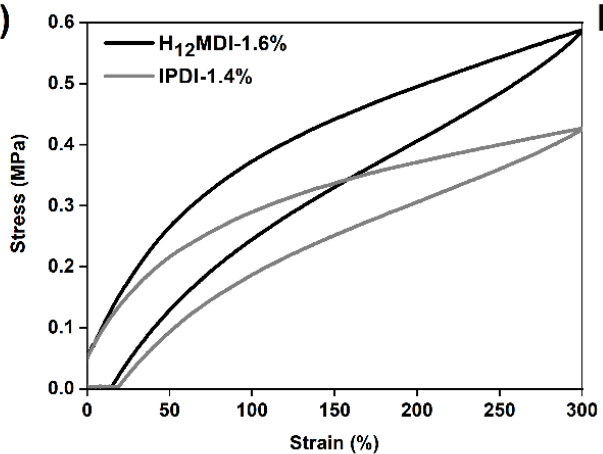

c)

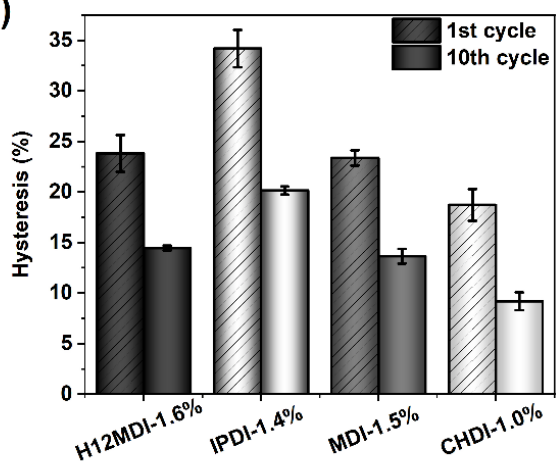

b)
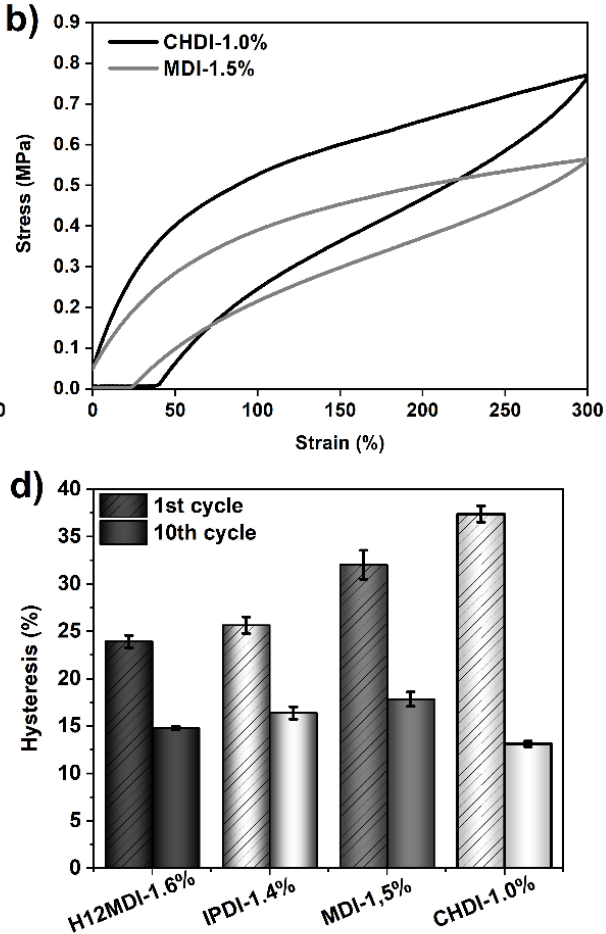

Figure 13. Results of tensile hysteresis measurements for PSU copolymers synthesized from the aliphatic diisocyanates $\mathrm{H}_{12} \mathrm{MDI}$, IPDI, and $\mathrm{CHDI}$, and from the aromatic diisocyanate MDI without chain extenders. 300\%-hysteresis curves (1st cycle) of the copolymers based on (a) $\mathrm{H}_{12} \mathrm{MDI}$ and IPDI and (b) CHDI and MDI. Mean hysteresis values of the copolymers at the 1st and 10th cycle at (c) $100 \%$ strain and (d) 300\% strain. The error bars represent the standard deviation of three repeated measurements. At 100\% strain, the highest hysteresis is observed for the IPDI-based copolymer. At $300 \%$ strain, the MDI- and CHDI-based copolymers exhibit the highest hysteresis values.

When the PSU copolymers are elongated to $300 \%$, another situation is apparent. The highest first-cycle hysteresis values are observed in the elastomers based on CHDI (37\%) and MDI (32\%) as the graphs in Figure 13d show. Furthermore, both elastomers show a higher instantaneous set, which indicates more effective hydrogen bonding within the hard domains owing to the higher order of the HSs [28]. However, the hysteresis values after the third cycle become quite similar, irrespective of diisocyanate structure. From the different hysteresis behavior of the copolymer containing the symmetric CHDI, it can be concluded that the ordered HSs are more densely packed due to strong hydrogen bonding interactions. This seems to partially protect the morphology from deformation at low strains. At high strains, however, the morphology of the crystalline hard domains is disrupted, leading to a pronounced increase in hysteresis.

Das et al. synthesized several PTMO-based non-chain-extended PUs und PUrs from diisocyanates of varying symmetry and studied their morphologies and mechanical properties [28]. Unlike the PU copolymers, all the urea copolymers displayed well-separated microphase morphologies with thread-like hard domains and showed high mechanical strengths combined with good elastomeric properties. When comparing the results of the first-cycle hysteresis at $300 \%$ strain, they found that the values increased in the order $\mathrm{H}_{12} \mathrm{MDI}<\mathrm{MDI}<\mathrm{CHDI}$. Furthermore, the instantaneous set was found to increase in the same order and was highest in the copolymer based on the symmetrical CHDI due to the breaking and restructuring of the crystalline hard domains [28]. A similar symmetry effect on the first-cycle hysteresis was reported by Prisacariu and Scortanu, who prepared polyether-based PUs from MDI and crystallizable DBDI [27]. 


\subsection{In Vitro Cytotoxicity}

All PSU elastomers and copolymers were subjected to in vitro cytotoxicity assessment to determine whether these polymers, which were synthesized from different diisocyanates with varying HS contents, have adverse effects on $\mathrm{HaCaT}$ cell viability. An aliphatic polycarbonate-based medical-grade PU (Carbothane PS 3585A) was selected as non-cytotoxic reference material.

As the cell proliferation results in Figure 14 show, the cell-medium extracts of the PSU elastomers and copolymers clearly do not contain any cytotoxic low-molecular-weight leachables. All the PSU extracts exhibit a cell proliferation of over $97 \%$ and thus are not cytotoxic. These results confirm that it is beneficial to apply amino-terminated monomers to the synthesis of elastomers because the high reactivity of these groups toward aliphatic diisocyanates prevents the formation of low-molecular-weight chains and, more importantly, ensures complete conversion of the isocyanate groups without the use of toxic organotin-based catalysts. As expected, the reference material Carbothane also shows no cytotoxic effects.
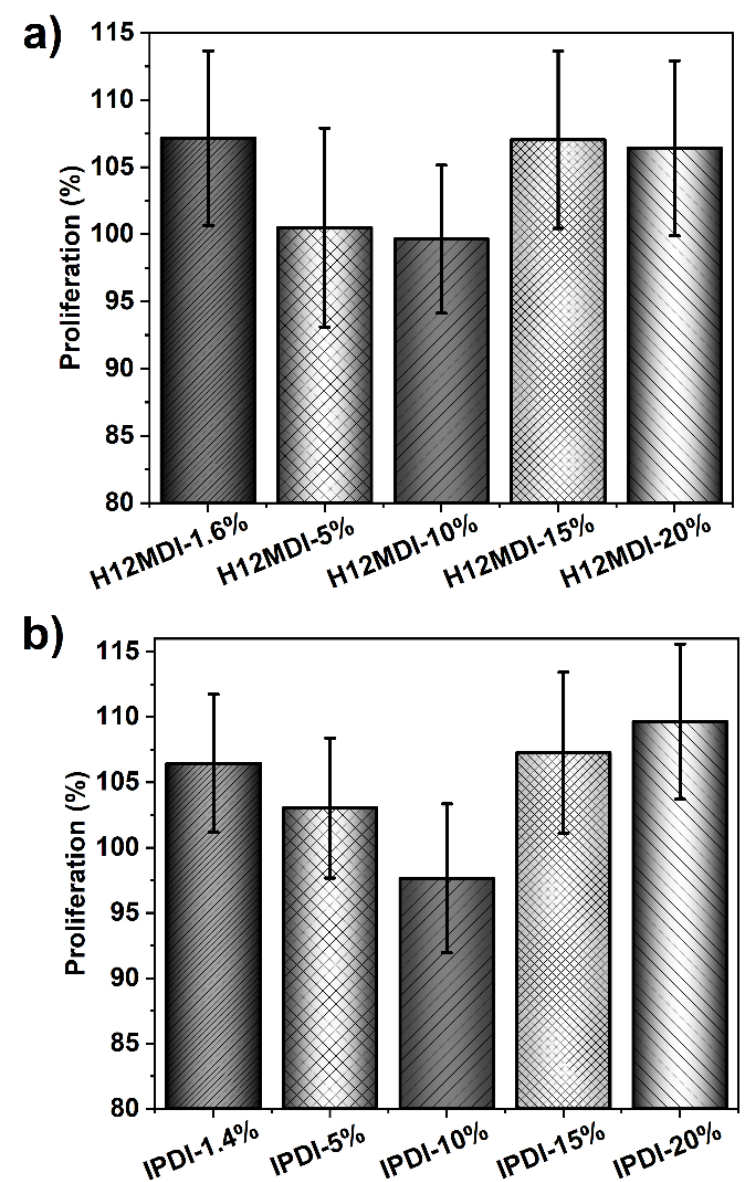

Figure 14. Cont. 


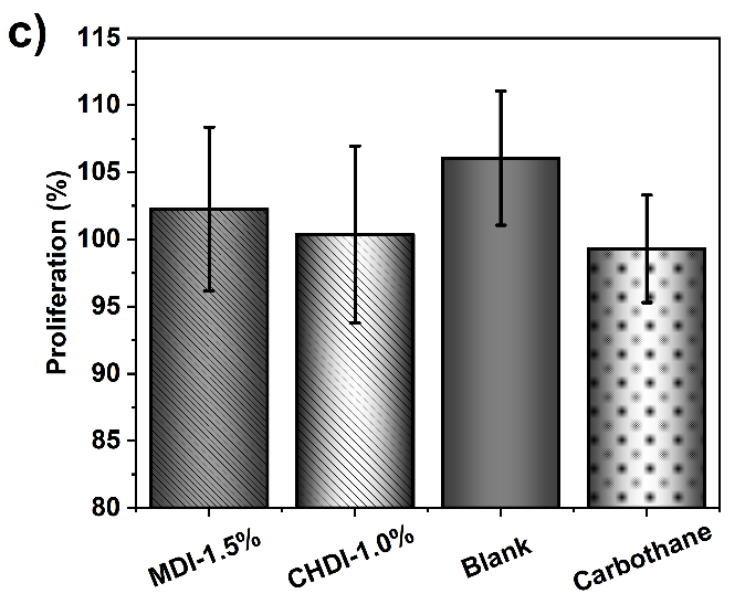

Figure 14. Viability of HaCaT cells after treatment with cell-medium extracts of PSU elastomers synthesized from (a) $\mathrm{H}_{12} \mathrm{MDI}$, (b) IPDI, and (c) PSU copolymers synthesized from MDI and CHDI without chain extenders. The blank represents cell medium without a sample subjected to the same extraction conditions as the PSU samples. A medical-grade PU (Carbothane PS-3585A) was chosen as a reference material. Cell viabilities, as determined using the MTS cell proliferation assay, for all PSUs, PSU copolymers, and Carbothane are all over 95\%. The error bars represent the standard deviation from 18 repeated measurements for each polymer-sample. Thus, no polymer extracts show any cytotoxic effects.

\section{Conclusions}

Three series of PDMS-based urea elastomers were prepared by two-step polyaddition reactions in THF solution. The main objectives were to investigate the influence of (i) HS content and (ii) diisocyanate chemical structure $\left(\mathrm{H}_{12} \mathrm{MDI}\right.$, IPDI, MDI, and $\mathrm{CHDI}$ ) on the transparencies and mechanical properties of the resultant PSU elastomers. Particularly, the effects of altered hard domain morphology upon increasing the content of urea groups on YM, UTS, and 10-cycle hysteresis behavior was interesting. For this reason, the HS contents in the PSUs based on IPDI and $\mathrm{H}_{12} \mathrm{MDI}$ were increased stepwise from $5 \%$ to $20 \%$ through incorporation of an increasing ratio of the diisocyanate and the chain extender APTMDS. Attempts to similarly increase the HS contents in the MDI- and CHDI-based elastomers resulted in tough and opaque polymer gels that were insoluble even in polar solvents and solvent mixtures.

Furthermore, the effects of diisocyanate structure on transparency and mechanical properties were studied on a series of non-chain-extended PSU copolymers, which were prepared with very low urea HS contents of $1.0-1.6 \%$.

Transparent PSU elastomers are obtained for HS contents below 10\%. Increasing opacity in the PSU films is observed for HS contents of $15 \%$ and higher. Transmittance at $750 \mathrm{~nm}$, which is the upper edge of the visible light region, decreases to $85 \%$ and $60 \%$ at HS contents of $15 \%$ and $20 \%$, respectively. This loss in transparency is attributed to light scattering by large thread-like hard domains that possibly form due to an increase in the HS molecular weight from 2900 to $4100 \mathrm{~g} \cdot \mathrm{mol}^{-1}$.

Independently of the diisocyanate structure and hence of the structure of the HSs, all the PSU copolymers exhibit transmittances of over $90 \%$ between 750 and $450 \mathrm{~nm}$, making them potentially suitable for optical applications such as soft contact lenses or intraocular lenses. Furthermore, the MDI-based copolymer shows a cut off at approximately $300 \mathrm{~nm}$ and, as a result, blocks UV-B radiation. This may be a beneficial property in applications where UV sensitive materials or biological structures need to be protected. Interestingly, though this elastomer contains an aromatic diisocyanate, the typical phenomenon of yellowing upon exposure to UV light is not observed for this elastomer.

There is a linear correlation of YM and UTS with HS content in the PSU elastomers based on $\mathrm{H}_{12}$ MDI. Conversely, values of YM in the IPDI series follow a logarithmical 
trend with increasing HS content. YM is highest for the CHDI-based copolymer due to the high symmetry of the diisocyanate, which allows a highly ordered and tightly packed HS structure. Conversely, the elastomer based on the asymmetric IPDI exhibits the lowest YM. The PSU copolymers based on $\mathrm{H}_{12} \mathrm{MDI}$ and MDI exhibit intermediate and similar YM values. All PSUs (except CHDI) exhibit high elasticity, displaying elongations between $760 \%$ and $1100 \%$.

The hysteresis values for the first cycle exhibited two different trends. At low HS contents (between $1.4 \%$ and $10 \%$ ), an increasing concentration of urea groups mechanically stabilizes the elastomer from the impact of strain, resulting in a reduction of hysteresis. It is presumed that the morphology in these elastomers consists of small, randomly distributed hard domains that physically reinforce the continuous PDMS soft phase by inter-urea hydrogen bonding. However, when the HS content is increased from $10 \%$ to $20 \%$, the first cycle hysteresis values increase. This effect was explained in terms of an altered hard domain morphology. Due to an increase in HS molecular weight, it is postulated that the hard domains increase in size and presumably form a more interpenetrating network of thread-like hard domains. Upon the impact of strain, these domains are distinctly deformed or disrupted, leading to a higher hysteresis in the first cycle. However, the hysteresis values remain nearly constant after the 3rd cycle, whether the HS content is $10 \%$ or more. As expected, a higher strain (300\% vs. $100 \%$ ) induces higher hysteresis values in the PSU elastomers and in the copolymers based on MDI and CHDI. Interestingly, the hysteresis values of the $\mathrm{H}_{12} \mathrm{MDI}$-copolymer are equal for both strains and, in the case of IPDI, slightly reduced. This may indicate that monodisperse urea HSs formed by the unsymmetric diisocyanates were already disrupted at low strain.

When comparing the results of mechanical characterization of all PSUs, an optimum balance between reasonable mechanical stability, elasticity, and low hysteresis is identified for the elastomers PSU $\mathrm{H}_{12} \mathrm{MDI}-10 \%$ and PSU CHDI-1.0\%.

None of the PSUs exhibit any cytotoxic effects on HaCaT cells, demonstrating their potential for use in biomedical applications.

Author Contributions: Conceptualization, N.R., A.K., G.E.M.T. and G.L.; methodology, N.R., L.K., A.K., G.E.M.T. and G.L.; validation, N.R., K.A., L.K. and T.G.; formal analysis, N.R., K.A., L.K. and G.L.; investigation, N.R., K.A., L.K. and T.G.; resources, A.K., G.E.M.T. and G.L.; writing-original draft preparation, N.R.; writing-review and editing, K.A., L.K., T.G., A.K., G.E.M.T. and G.L.; visualization, N.R.; supervision, A.K., G.E.M.T. and G.L.; project administration, N.R. and G.L.; funding acquisition, G.E.M.T. and G.L. All authors have read and agreed to the published version of the manuscript.

Funding: This research was funded by the Federal Ministry of Education and Research (BMBF), grant number 13FH032I3. The article processing charge was funded by the Baden-Württemberg Ministry of Science, Research and Culture in the funding program Open Access Publishing.

Institutional Review Board Statement: Not applicable.

Informed Consent Statement: Not applicable.

Data Availability Statement: Data are available at Reutlingen University.

Acknowledgments: The authors would like to express thanks to Herbert Thelen and André Lemme from Biotronik for performing ethylene oxide sterilization of the PSU elastomers and copolymers. Natascha Riehle thanks Angelika Dürney from Reutlingen University for her guidance, advice, and help in cell cultivation. Furthermore, Natascha Riehle thanks Immanuel Trick from Reutlingen University for his support in preparing trans-1,4-cyclohexane dicarboxylic acid for CHDI-synthesis and Sheila Mahler from Reutlingen University for her support with stress-strain, hysteresis, and viscosity measurements.

Conflicts of Interest: The authors declare no conflict of interest. 
Appendix A. Synthesis of Polydimethylsiloxane-Based PUr Elastomers Comprising Varying Hard Segment Contents and Different Diisocyanates
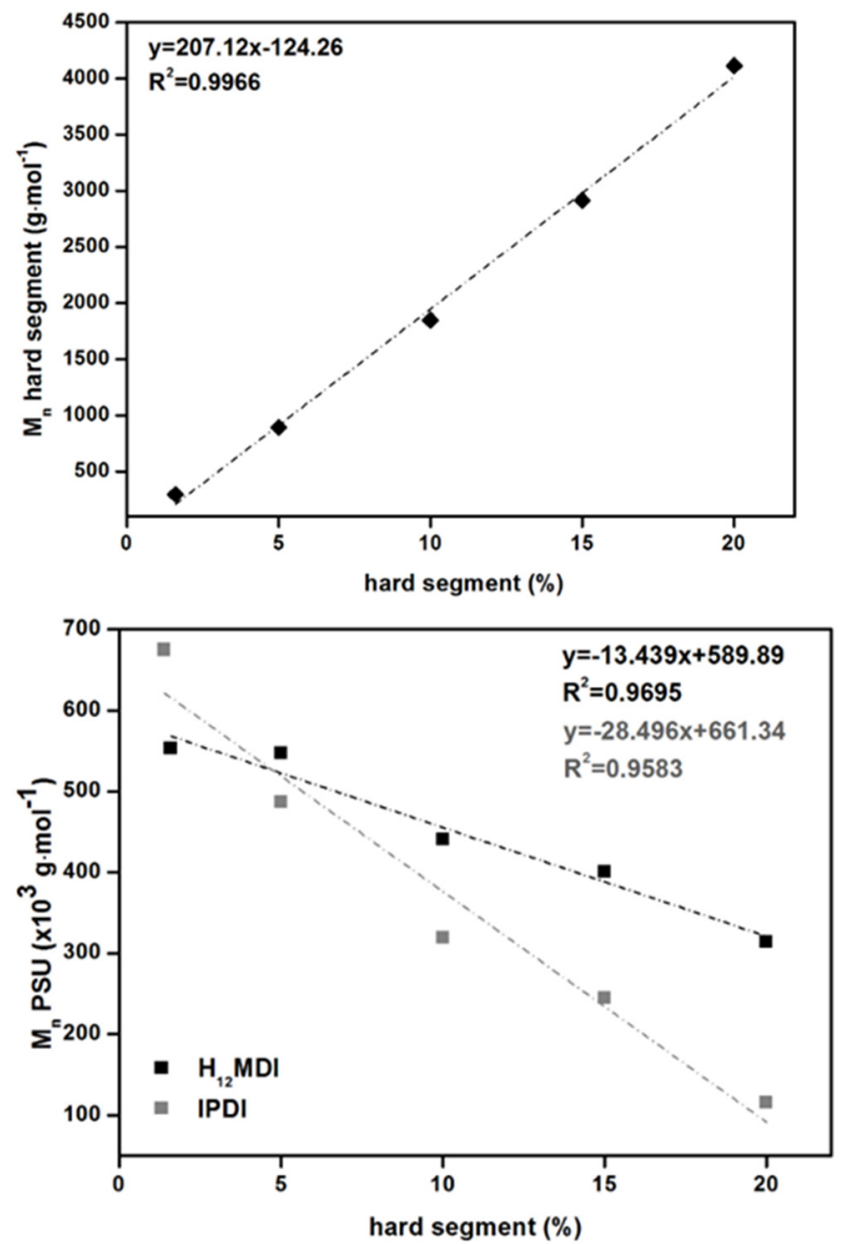

Figure A1. (top) Calculated average hard segment (HS) molecular weights as a function of HS content in PSU elastomers synthesized from $\mathrm{H}_{12} \mathrm{MDI}$. (bottom) Number average molecular weights of PSU elastomers synthesized from $\mathrm{H}_{12} \mathrm{MDI}$ (black squares) and IPDI (grey squares) determined by SEC as a function of HS content.

\section{Appendix B. Determination of Intrinsic Viscosity of the Polydimethylsiloxane-Based} PUr Elastomers

Intrinsic viscosity of the PDMS-based polyurea-elastomers was determined using an Ubbelohde viscometer. PSU solutions comprising four concentrations $\left(0.5-2.0 \mathrm{~g} \cdot \mathrm{dL}^{-1}\right)$ were prepared in THF and flow times for each polymer solution were measured (10 replicates) relative to the solvent to calculate the specific viscosity $\left(\eta_{\text {spec. }}\right)$ according to Equation (A1).

$$
\eta_{\text {spec. }}=\frac{\eta_{P S}-\eta_{\text {solvent }}}{\eta_{\text {solvent }}} \equiv \eta_{\text {spec. }}=\frac{t_{P S}-t_{\text {solvent }}}{t_{\text {solvent }}}
$$

were $t_{P S}$ and $t_{\text {solvent }}$ are the measured times (s) of a defined volume of the polymer solution/solvent passing the capillary.

The reduced viscosity $\eta_{\text {red. }}$ is obtained, when dividing $\eta_{\text {spec. }}$ by the concentration of the polymer solution.

$$
\eta_{\text {red. }}=\frac{\eta_{\text {spec }}}{c}
$$


The intrinsic viscosity $[\eta]$ is a measure of the capability of a polymer in solution to increase the viscosity of the solution and is obtained when extrapolating $\eta_{\text {spec }}$, to a concentration of zero.

$$
[\eta]=\lim _{n \rightarrow 0} \frac{\eta_{\text {spec. }}}{c}
$$

According to the relation of reduced viscosity and intrinsic viscosity, which was described by Huggins, $[\eta]$ can be calculated as intercept of a fitting curve where reduced viscosity is plotted against the polymer concentration within the solution. $k_{2}$ is called Huggins viscosity constant and is a measure of the solvent quality, i.e., if the selected solvent is a good or poor solvent for the polymer [66].

$$
\eta_{\text {red. }}=\frac{\eta_{\text {spec. }}}{c}=[\eta]+k_{2}[\eta]^{2} c
$$

The Mark-Houwink-Sakurada equation relates the intrinsic viscosity $[\eta]$ to the viscosity-average molecular weight $\bar{M}_{v}$ of a polymer. If the parameters $K$ and $\alpha$ (for a specific polymer-solvent system) are known, one can approximately calculate the weightaverage molecular weight $\bar{M}_{w}$ of the polymer.

$$
[\eta]=K \cdot M_{v}^{\alpha}
$$

In case of polymer-solvent systems, where the Mark-Houwink parameters are not reported, the parameters can be calculated by plotting the logarithm of intrinsic viscosity against the logarithm of molecular weight. The parameters $K$ and $\alpha$ are derived as intercept and slope of the linear curve.

$$
\log [\eta]=\log K+\alpha \cdot \log M
$$

a)

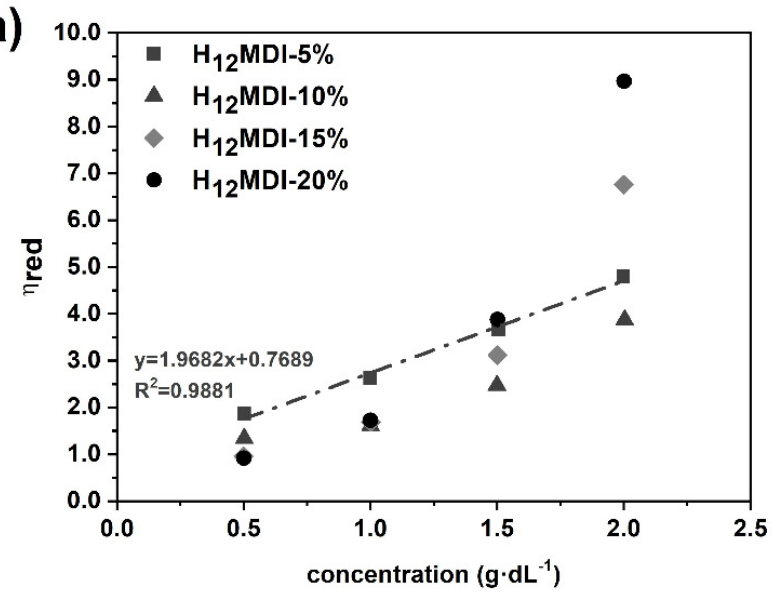

b)

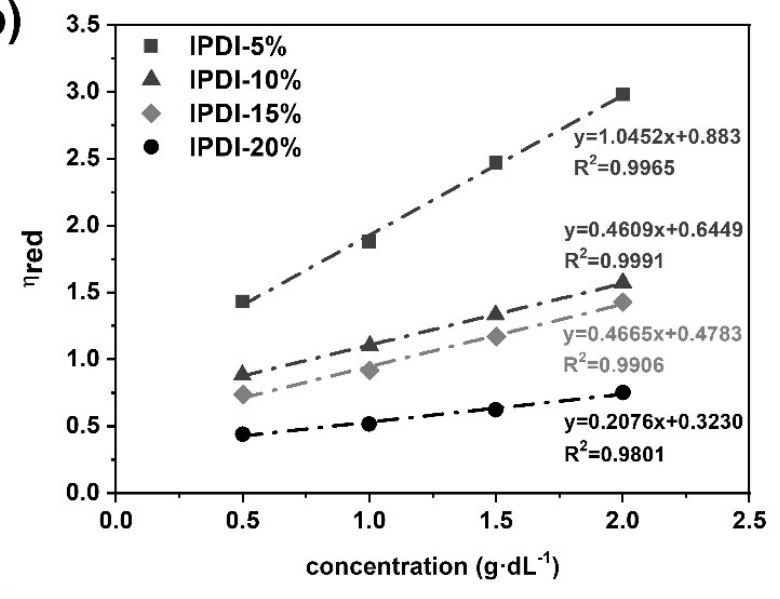

Figure A2. Cont. 


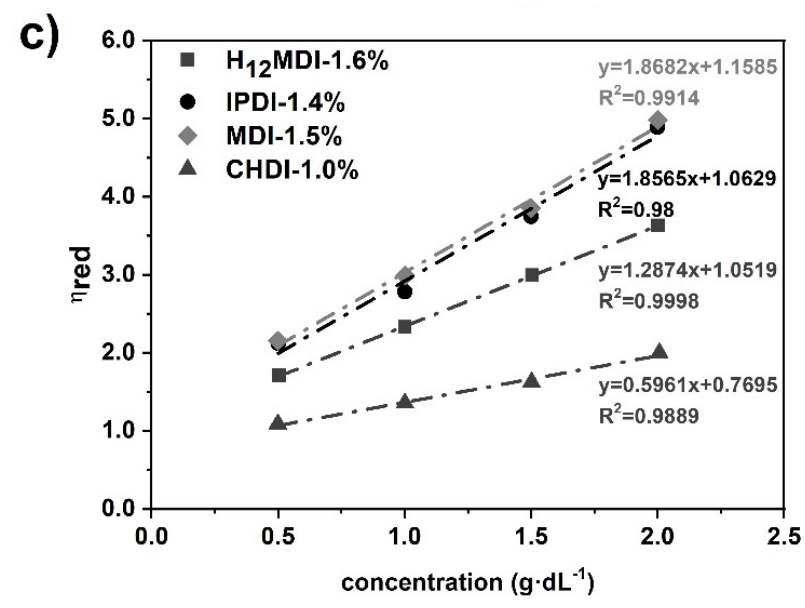

Figure A2. Huggins plots of reduced viscosity $\left(\eta_{\text {red. }}\right)$ versus concentration $\left(\mathrm{g} \cdot \mathrm{dL}^{-1}\right)$ of four PSU solutions in THF. The intrinsic viscosity $[\eta]$ was calculated by extrapolation of the Huggins fitting curves. (a) Huggins plots of the PSU $\mathrm{H}_{12} \mathrm{MDI}$ elastomers comprising HS contents between $5 \%$ and $20 \%$. A linear fitting curve was only obtained for the elastomer comprising $5 \%$ HS content. Values of reduced viscosity of the PSU $\mathrm{H}_{12} \mathrm{MDI}$ elastomers containing higher HS contents behaved not linear and seemed to rather follow an exponential trend. (b) Huggins plots of the PSU IPDI elastomers containing HS contents between 5\% and 20\%. (c) Huggins plots of PSU copolymers that were synthesized without chain extender.

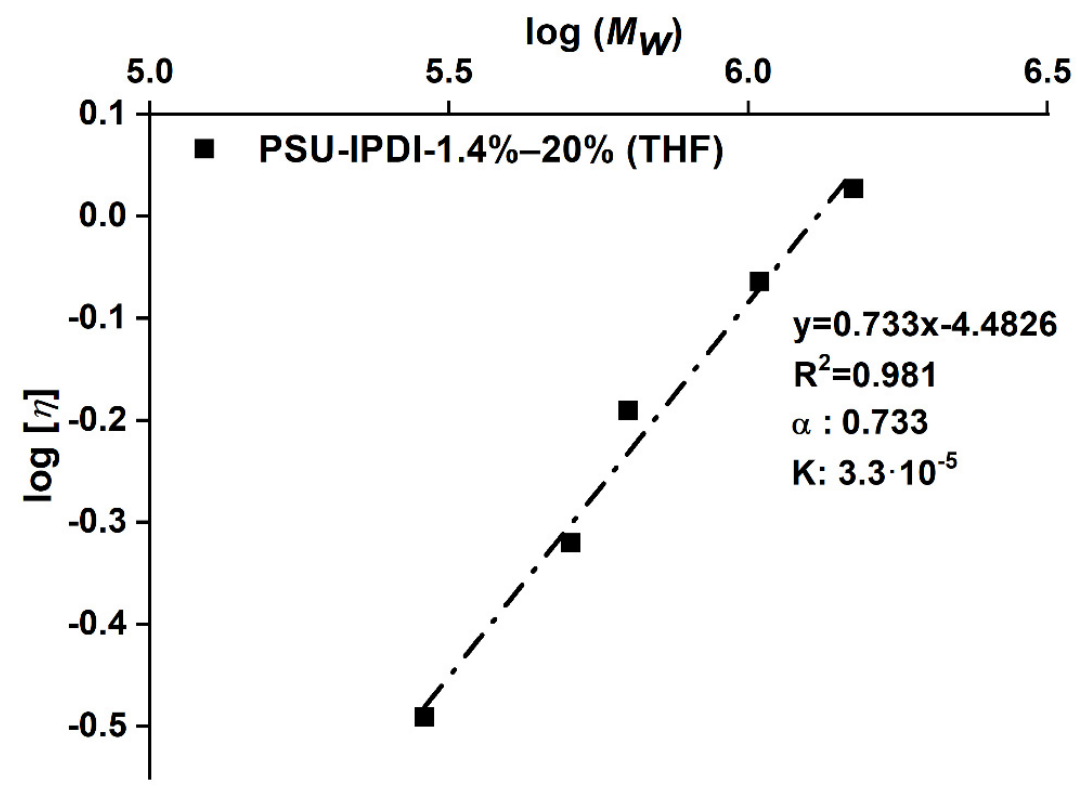

Figure A3. Double logarithmic plot of intrinsic viscosity $[\eta]$ versus weight average molecular weight $\bar{M}_{w}$ of the PSU IPDI elastomers determined from SEC. The Mark-Houwink parameters $\alpha$ and $K$ were obtained as slope and intercept from the linear fitting curve, and were 0.733 and $3.3 \times 10^{-5}$, respectively. 
Appendix C. Synthesis of Polydimethylsiloxane-Based PUr Elastomers Followed by ATR FTIR Spectroscopy
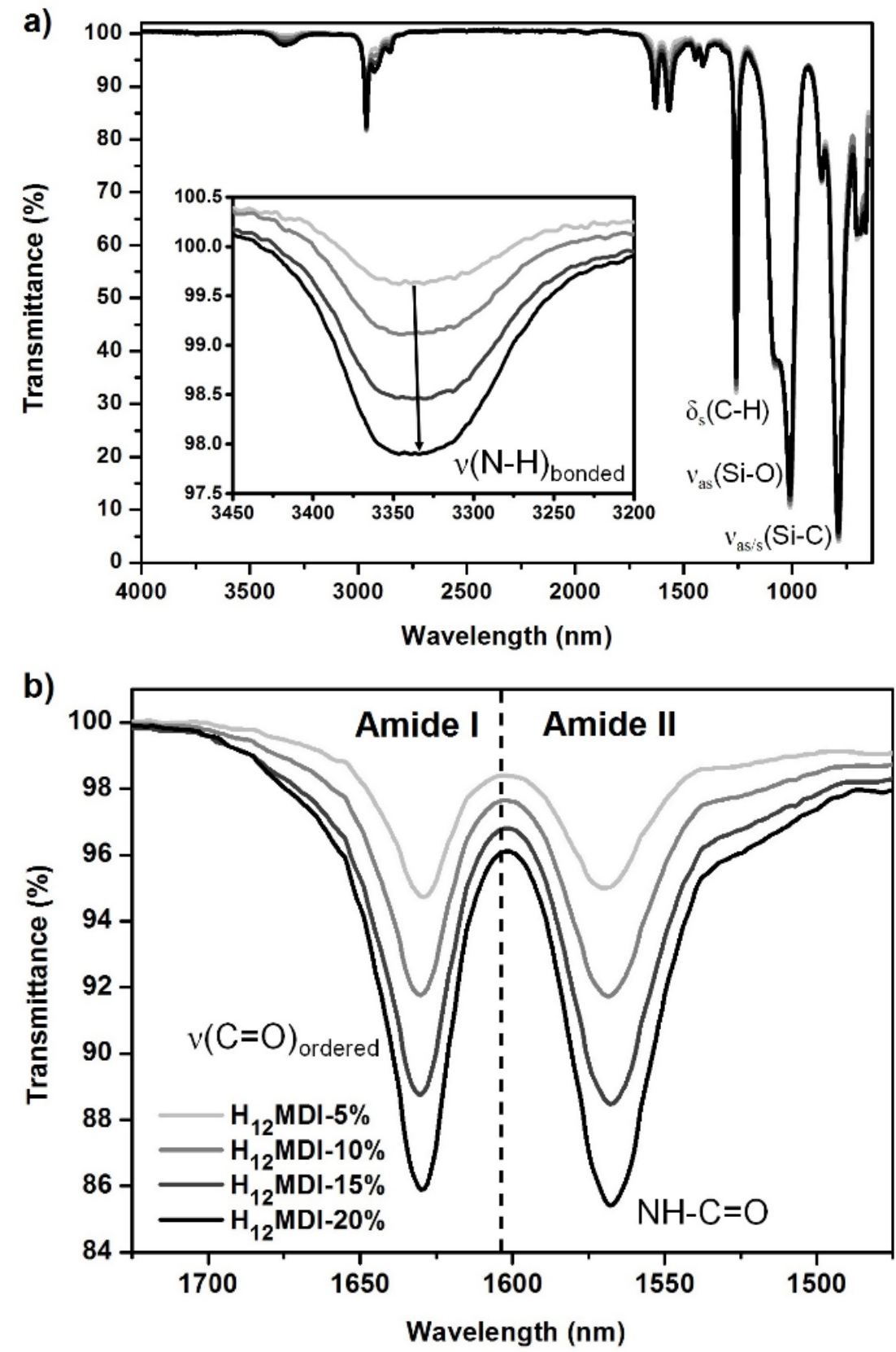

Figure A4. ATR-FTIR spectra of PDMS-based polyurea elastomers synthesized from $\mathrm{H}_{12} \mathrm{MDI}$ with increasing HS contents (5-20\%). (a) The enlarged N-H stretching region at 3450-3200 $\mathrm{cm}^{-1}$ shows that all urea groups were involved in hydrogen bonding interactions. The bands below $1500 \mathrm{~cm}^{-1}$ correspond to the PDMS soft segment vibrations at: $1258 \mathrm{~cm}^{-1}\left(\delta_{\mathrm{s}} \mathrm{C}-\mathrm{H}\right.$ in $\left.\mathrm{CH}_{3}\right), 1010 \mathrm{~cm}^{-1}$ ( $\left.v_{\text {as }} \mathrm{Si}-\mathrm{O}\right)$, $864 \mathrm{~cm}^{-1}\left(\delta_{\text {as }} \mathrm{C}-\mathrm{H}\right.$ in Si- $\left.\left(\mathrm{CH}_{3}\right)_{2}\right)$, and $786 \mathrm{~cm}^{-1} / 702 \mathrm{~cm}^{-1}\left(v_{\mathrm{as}} / \mathrm{s} \mathrm{Si}-\mathrm{C}\right.$ in Si- $\left.\left(\mathrm{CH}_{3}\right)_{2}\right)$. (b) Enlarged region of the Amide I ( $\mathrm{C}=\mathrm{O}$ stretching vibration) at $1630 \mathrm{~cm}^{-1}$ and Amide II $(\vee \mathrm{C}-\mathrm{N}+\delta \mathrm{N}-\mathrm{H})$ vibrations at $1568 \mathrm{~cm}^{-1}$. The band positions of the $\mathrm{C}=\mathrm{O}$ stretching vibrations indicate that all urea groups within the elastomers were hydrogen-bonded in an ordered manner $[45,67,68]$. 
Appendix D. Transparency of Novel Films as Documented by Photographs of Polydimethylsiloxane-Based PUr Elastomer Films

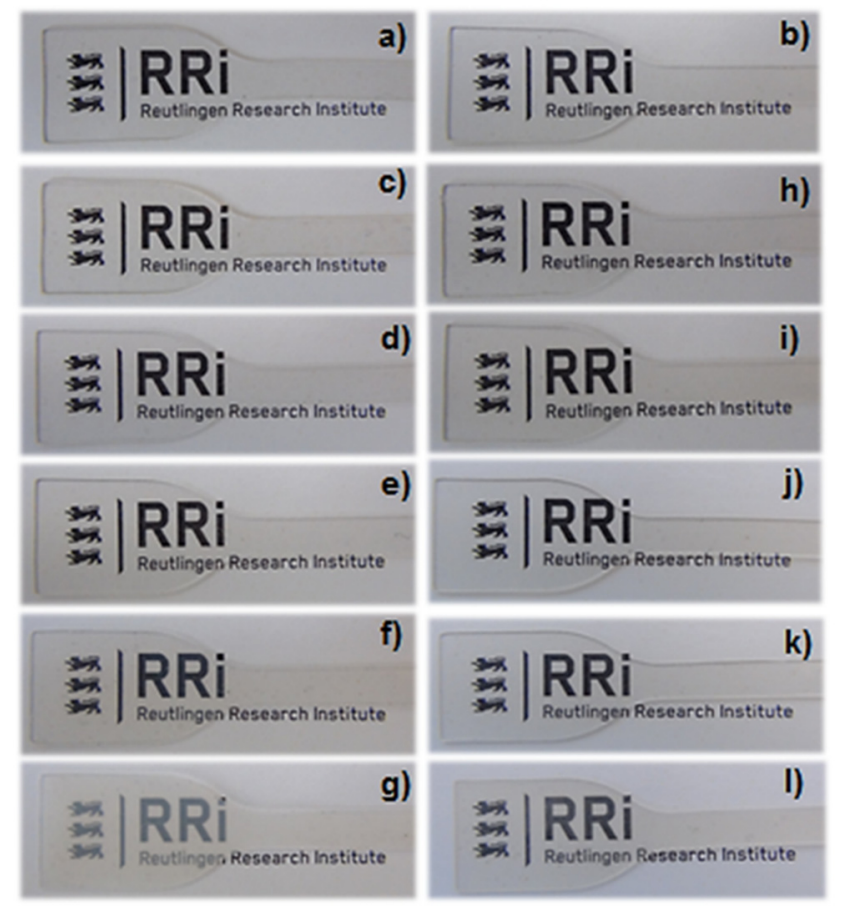

Figure A5. Photographs of PDMS-based PUr elastomer samples used for tensile testing. (a) PSU MDI-1.5\%. (b) PSU CHDI-1.0\%. (c-g) PSU H ${ }_{12}$ MDI series with HS contents: (c) $1.6 \%$, (d) 5\%, (e) 10\%, (f) $15 \%$, and (g) 20\%. (h-l) PSU IPDI series with HS contents: (h) 1.4\%, (i) 5\%, (j) 10\%, (k) 15\%, and (l) $20 \%$. All PSU elastomer films were transparent until a HS content of $10 \%$. The PSU elastomer films became translucent at HS contents of $15 \%$ and $20 \%$.

\section{Appendix E. Mechanical Properties of Polydimethylsiloxane-Based PUr Elastomers Comprising Varying HS Contents and Different Diisocyanates}

Table A1. Young's modulus, ultimate tensile strength, and elongation at break of PDMS-based polyurea elastomers synthesized from the aliphatic diisocyanates $\mathrm{H}_{12} \mathrm{MDI}$, IPDI, and CHDI, and from the aromatic diisocyanate MDI. Values are given as an average of five repeated measurements \pm standard deviation.

\begin{tabular}{lccc}
\hline \multicolumn{1}{c}{ PSU } & $\begin{array}{c}\text { Young's } \\
\text { Modulus } \\
\mathbf{( M P a )}\end{array}$ & $\begin{array}{c}\text { Ultimate Tensile } \\
\text { Strength } \\
\mathbf{( M P a )}\end{array}$ & $\begin{array}{c}\text { Elongation } \\
\text { at Break } \\
\mathbf{( \% )}\end{array}$ \\
\hline PSU H $_{12}$ MDI-1.6\% & $0.52 \pm 0.05$ & $1.34 \pm 0.13$ & $836 \pm 137$ \\
PSU H $_{12}$ MDI-5\% & $0.83 \pm 0.07$ & $2.41 \pm 0.14$ & $902 \pm 51$ \\
PSU H $_{12}$ MDI-10\% & $0.96 \pm 0.13$ & $4.07 \pm 0.16$ & $922 \pm 103$ \\
PSU H $_{12}$ MDI-15\% & $1.17 \pm 0.07$ & $5.37 \pm 0.40$ & $814 \pm 86$ \\
PSU H $_{12}$ MDI-20\% & $1.34 \pm 0.06$ & $6.70 \pm 0.52$ & $758 \pm 93$ \\
PSU IPDI-1.4\% & $0.27 \pm 0.01$ & $1.00 \pm 0.07$ & $1080 \pm 84$ \\
PSU IPDI-5\% & $0.60 \pm 0.04$ & $1.88 \pm 0.13$ & $888 \pm 47$ \\
PSU IPDI-10\% & $0.92 \pm 0.04$ & $3.04 \pm 0.19$ & $880 \pm 101$ \\
PSU IPDI-15\% & $1.12 \pm 0.03$ & $4.56 \pm 0.38$ & $976 \pm 87$ \\
PSU IPDI-20\% & $1.28 \pm 0.10$ & $4.76 \pm 0.50$ & $816 \pm 108$ \\
PSU MDI-1.5\% & $0.48 \pm 0.04$ & $1.36 \pm 0.25$ & $1050 \pm 140$ \\
PSU CHDI-1.0\% & $0.91 \pm 0.05$ & $1.19 \pm 0.12$ & $602 \pm 77$ \\
\hline
\end{tabular}



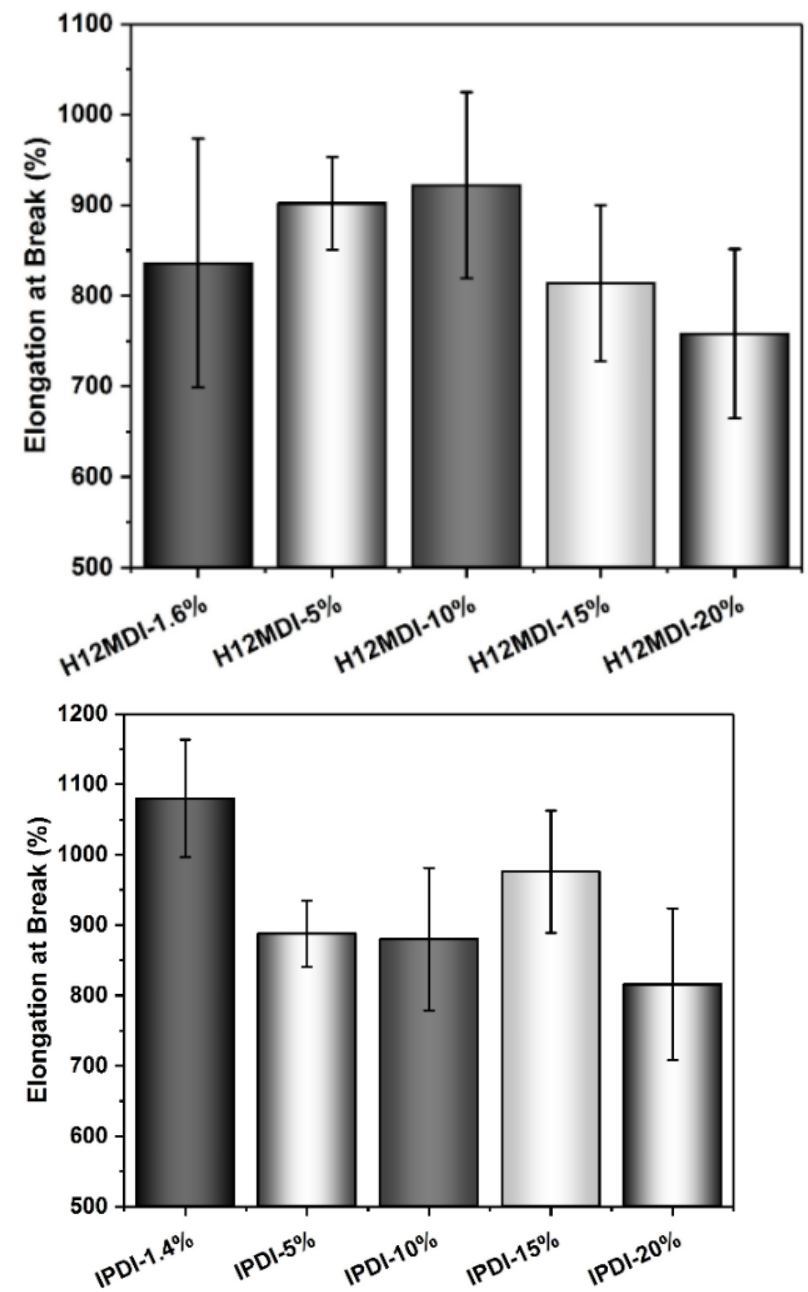

Figure A6. Elongation at break of PDMS-based polyurea-elastomers synthesized from $\mathrm{H}_{12} \mathrm{MDI}$ (top) and IPDI (bottom) comprising HS contents between $1.6 \%(1.4 \%)$ and $20 \%$.

Table A2. Tensile hysteresis at 100\% strain of PDMS-based polyurea elastomers synthesized from 4,4-methylenebis(cyclohexyl isocyanate) ( $\mathrm{H}_{12} \mathrm{MDI}$ ) with $\mathrm{HS}$ contents between $1.6 \%$ and $20 \%$. Values are given as an average of three repeated measurements. SD: standard deviation.

\begin{tabular}{ccccccccccc}
\hline PSU/Cycle & $\mathbf{1}$ & $\mathbf{2}$ & $\mathbf{3}$ & $\mathbf{4}$ & $\mathbf{5}$ & $\mathbf{6}$ & $\mathbf{7}$ & $\mathbf{8}$ & $\mathbf{9}$ & $\mathbf{1 0}$ \\
\hline $\mathrm{H}_{12} \mathrm{MDI}-1.6 \%$ & 23.8 & 16.4 & 15.5 & 15.1 & 14.7 & 14.7 & 14.6 & 14.5 & 14.4 & 14.5 \\
SD & 1.8 & 0.2 & 0.2 & 0.3 & 0.2 & 0.2 & 0.2 & 0.2 & 0.2 & 0.2 \\
$\mathrm{H}_{12} \mathrm{MDI}-5 \%$ & 11.2 & 6.9 & 6.1 & 5.7 & 5.5 & 5.3 & 5.3 & 5.2 & 5.0 & 5.0 \\
$\mathrm{SD}$ & 0.8 & 0.3 & 0.3 & 0.4 & 0.4 & 0.4 & 0.4 & 0.4 & 0.3 & 0.4 \\
$\mathrm{H}_{12} \mathrm{MDI}-10 \%$ & 8.8 & 3.7 & 3.3 & 3.0 & 2.9 & 2.8 & 2.7 & 2.7 & 2.7 & 2.6 \\
$\mathrm{SD}$ & 1.7 & 0.4 & 0.3 & 0.3 & 0.3 & 0.3 & 0.2 & 0.3 & 0.3 & 0.3 \\
$\mathrm{H}_{12} \mathrm{MDI}-15 \%$ & 12.3 & 3.6 & 3.2 & 3.0 & 2.8 & 2.8 & 2.7 & 2.7 & 2.6 & 2.6 \\
$\mathrm{SD}$ & 4.7 & 0.2 & 0.1 & 0.1 & 0.1 & 0.1 & 0.1 & 0.1 & 0.1 & 0.1 \\
$\mathrm{H}_{12} \mathrm{MDI}-20 \%$ & 27.8 & 5.1 & 4.5 & 4.3 & 4.2 & 4.1 & 4.1 & 4.0 & 4.0 & 4.0 \\
SD & 3.5 & 1.3 & 1.4 & 1.5 & 1.5 & 1.5 & 1.5 & 1.6 & 1.5 & 1.5
\end{tabular}


Table A3. Tensile hysteresis at 100\% strain of PDMS-based polyurea elastomers synthesized from isophorone diisocyanate (IPDI) with HS contents between $1.4 \%$ and $20 \%$. Values are given as an average of three repeated measurements. SD: standard deviation.

\begin{tabular}{ccccccccccc}
\hline PSU/Cycle & $\mathbf{1}$ & $\mathbf{2}$ & $\mathbf{3}$ & $\mathbf{4}$ & $\mathbf{5}$ & $\mathbf{6}$ & $\mathbf{7}$ & $\mathbf{8}$ & $\mathbf{9}$ & $\mathbf{1 0}$ \\
\hline IPDI-1.4\% & 34.2 & 20.9 & 20.3 & 20.1 & 20.0 & 19.9 & 19.9 & 20.0 & 20.1 & 20.1 \\
SD & 1.8 & 0.7 & 0.7 & 0.6 & 0.5 & 0.4 & 0.5 & 0.5 & 0.6 & 0.4 \\
IPDI-5\% & 17.0 & 10.3 & 9.4 & 9.1 & 8.8 & 8.7 & 8.5 & 8.5 & 8.4 & 8.3 \\
SD & 0.3 & 0.6 & 0.7 & 0.7 & 0.8 & 0.8 & 0.8 & 0.8 & 0.8 & 0.8 \\
IPDI-10\% & 9.5 & 4.6 & 4.0 & 3.7 & 3.4 & 3.3 & 3.3 & 3.2 & 3.1 & 3.1 \\
SD & 0.6 & 0.3 & 0.3 & 0.3 & 0.3 & 0.2 & 0.2 & 0.2 & 0.2 & 0.2 \\
IPDI-15\% & 13.2 & 5.1 & 4.4 & 4.0 & 3.9 & 3.7 & 3.7 & 3.6 & 3.5 & 3.4 \\
SD & 0.4 & 0.1 & 0.1 & 0.1 & 0.0 & 0.1 & 0.1 & 0.1 & 0.1 & 0.1 \\
IPDI-20\% & 21.6 & 7.9 & 6.8 & 6.3 & 6.0 & 5.8 & 5.6 & 5.5 & 5.4 & 5.3 \\
SD & 0.6 & 0.3 & 0.2 & 0.2 & 0.2 & 0.2 & 0.2 & 0.2 & 0.2 & 0.2 \\
\hline
\end{tabular}

Table A4. Tensile hysteresis at 100\% strain of PDMS-based polyurea copolymers synthesized from 4,4'-methylenebis(phenyl isocyanate) (MDI) and trans-1,4-cyclohexane diisocyanate (CHDI) with urea HSs of approximately $1.5 \%$ and $1.0 \%$. Values are given as an average of three repeated measurements. SD: standard deviation.

\begin{tabular}{ccccccccccc}
\hline PSU/Cycle & $\mathbf{1}$ & $\mathbf{2}$ & $\mathbf{3}$ & $\mathbf{4}$ & $\mathbf{5}$ & $\mathbf{6}$ & $\mathbf{7}$ & $\mathbf{8}$ & $\mathbf{9}$ & $\mathbf{1 0}$ \\
\hline MDI-1.5\% & 23.4 & 15.7 & 15.1 & 14.6 & 14.3 & 14.2 & 14.1 & 13.9 & 13.8 & 13.7 \\
SD & 0.8 & 0.7 & 0.7 & 0.6 & 0.8 & 0.9 & 0.9 & 0.7 & 0.8 & 0.7 \\
CHDI-1.0\% & 18.7 & 12.3 & 11.1 & 10.5 & 10.1 & 9.9 & 9.7 & 9.5 & 9.3 & 9.2 \\
SD & 1.6 & 0.7 & 0.7 & 0.8 & 0.9 & 0.8 & 0.8 & 0.8 & 0.9 & 0.9 \\
\hline
\end{tabular}

Table A5. Tensile hysteresis at 300\% strain of PDMS-based polyurea elastomers synthesized from 4,4-methylenebis(cyclohexyl isocyanate) $\left(\mathrm{H}_{12} \mathrm{MDI}\right.$ ) with $\mathrm{HS}$ contents between $1.6 \%$ and $20 \%$. Values are given as an average of three repeated measurements. SD: standard deviation.

\begin{tabular}{ccccccccccc}
\hline PSU/Cycle & $\mathbf{1}$ & $\mathbf{2}$ & $\mathbf{3}$ & $\mathbf{4}$ & $\mathbf{5}$ & $\mathbf{6}$ & $\mathbf{7}$ & $\mathbf{8}$ & $\mathbf{9}$ & $\mathbf{1 0}$ \\
\hline $\mathrm{H}_{12} \mathrm{MDI}-1.6 \%$ & 23.9 & 17.0 & 15.8 & 15.4 & 15.2 & 15.0 & 14.9 & 14.8 & 14.8 & 14.8 \\
$\mathrm{SD}$ & 0.6 & 0.3 & 0.2 & 0.2 & 0.2 & 0.2 & 0.2 & 0.2 & 0.2 & 0.2 \\
$\mathrm{H}_{12} \mathrm{MDI}-5 \%$ & 21.4 & 12.1 & 10.8 & 10.2 & 9.9 & 9.6 & 9.4 & 9.2 & 9.1 & 8.9 \\
$\mathrm{SD}$ & 1.1 & 0.2 & 0.3 & 0.3 & 0.3 & 0.3 & 0.3 & 0.3 & 0.3 & 0.3 \\
$\mathrm{H}_{12} \mathrm{MDI}-10 \%$ & 21.1 & 9.5 & 8.4 & 7.9 & 7.5 & 7.3 & 7.1 & 6.9 & 6.8 & 6.7 \\
$\mathrm{SD}$ & 1.3 & 1.3 & 1.2 & 1.2 & 1.2 & 1.1 & 1.1 & 1.1 & 1.0 & 1.0 \\
$\mathrm{H}_{12} \mathrm{MDI}-15 \%$ & 28.9 & 10.6 & 9.2 & 8.6 & 8.1 & 7.9 & 7.6 & 7.4 & 7.3 & 7.1 \\
$\mathrm{SD}$ & 1.8 & 0.9 & 0.8 & 0.8 & 0.8 & 0.7 & 0.7 & 0.7 & 0.6 & 0.7 \\
$\mathrm{H}_{12} \mathrm{MDI}-20 \%$ & 27.5 & 10.0 & 8.7 & 8.2 & 7.8 & 7.6 & 7.3 & 7.2 & 7.1 & 7.0 \\
$\mathrm{SD}$ & 0.9 & 0.3 & 0.3 & 0.5 & 0.4 & 0.4 & 0.3 & 0.3 & 0.4 & 0.5 \\
\hline
\end{tabular}

Table A6. Tensile hysteresis at 300\% strain of PDMS-based polyurea elastomers synthesized from isophorone diisocyanate (IPDI) with HS contents between $1.4 \%$ and $20 \%$. Values are given as an average of three repeated measurements. SD: standard deviation.

\begin{tabular}{ccccccccccc}
\hline PSU/Cycle & $\mathbf{1}$ & $\mathbf{2}$ & $\mathbf{3}$ & $\mathbf{4}$ & $\mathbf{5}$ & $\mathbf{6}$ & $\mathbf{7}$ & $\mathbf{8}$ & $\mathbf{9}$ & $\mathbf{1 0}$ \\
\hline IPDI-1.4\% & 25.6 & 18.1 & 17.2 & 16.8 & 16.6 & 16.5 & 16.4 & 16.4 & 16.3 & 16.4 \\
SD & 0.9 & 0.3 & 0.5 & 0.5 & 0.5 & 0.6 & 0.6 & 0.6 & 0.7 & 0.7 \\
IPDI-5\% & 26.9 & 14.5 & 13.1 & 12.3 & 11.9 & 11.6 & 11.4 & 11.2 & 11.1 & 10.9 \\
SD & 2.1 & 0.8 & 0.9 & 0.9 & 0.9 & 1.0 & 1.0 & 1.0 & 1.0 & 1.0 \\
IPDI-10\% & 25.0 & 10.0 & 8.7 & 8.1 & 7.8 & 7.5 & 7.3 & 7.2 & 7.0 & 6.9 \\
SD & 0.9 & 0.2 & 0.2 & 0.3 & 0.3 & 0.3 & 0.4 & 0.4 & 0.4 & 0.4 \\
IPDI-15\% & 30.7 & 11.2 & 9.6 & 9.0 & 8.6 & 8.3 & 8.1 & 7.9 & 7.7 & 7.6 \\
SD & 1.7 & 0.1 & 0.1 & 0.1 & 0.1 & 0.1 & 0.1 & 0.1 & 0.1 & 0.2 \\
IPDI-20\% & 41.5 & 13.4 & 11.5 & 10.6 & 10.0 & 9.7 & 9.4 & 9.2 & 9.0 & 8.8 \\
SD & 0.9 & 0.2 & 0.1 & 0.1 & 0.1 & 0.1 & 0.1 & 0.1 & 0.1 & 0.2 \\
\hline
\end{tabular}


Table A7. Tensile hysteresis at 300\% strain of PDMS-based polyurea copolymers synthesized from 4,4'-methylenebis(phenyl isocyanate) (MDI) and trans-1,4-cyclohexane diisocyanate (CHDI) with urea HSs of approximately $1.5 \%$ and $1.0 \%$. Values are given as an average of three repeated measurements. SD: standard deviation.

\begin{tabular}{ccccccccccc}
\hline PSU/Cycle & $\mathbf{1}$ & $\mathbf{2}$ & $\mathbf{3}$ & $\mathbf{4}$ & $\mathbf{5}$ & $\mathbf{6}$ & $\mathbf{7}$ & $\mathbf{8}$ & $\mathbf{9}$ & $\mathbf{1 0}$ \\
\hline MDI-1.5\% & 32.0 & 22.1 & 19.9 & 19.1 & 18.8 & 18.5 & 18.3 & 18.1 & 17.9 & 17.8 \\
SD & 1.5 & 1.1 & 0.6 & 0.7 & 0.7 & 0.7 & 0.7 & 0.6 & 0.7 & 0.8 \\
CHDI-1.0\% & 37.4 & 20.6 & 17.9 & 16.6 & 15.9 & 15.3 & 14.7 & 14.3 & 13.8 & 13.1 \\
SD & 0.9 & 0.5 & 0.5 & 0.4 & 0.3 & 0.3 & 0.3 & 0.3 & 0.3 & 0.3 \\
\hline
\end{tabular}
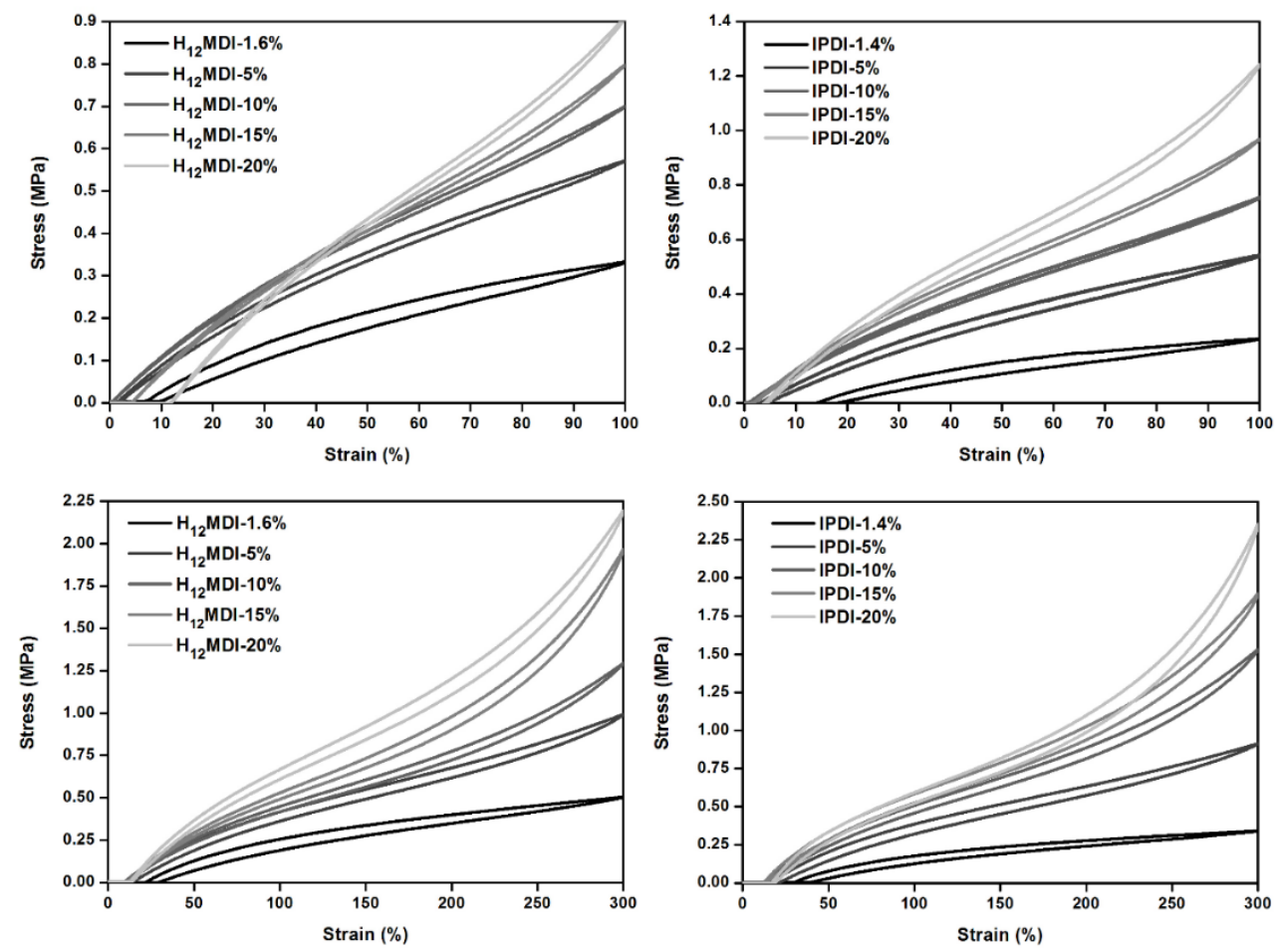

Figure A7. (top) 10th cycle hysteresis at 100\% strain of PDMS-based polyurea-elastomers synthesized from $\mathrm{H}_{12} \mathrm{MDI}$ and IPDI comprising different HS contents from $1.6 \%$ (1.4\%) to $20 \%$. (bottom) 10 th cycle hysteresis at $300 \%$ strain.

\section{Appendix F. Film Thicknesses of Polydimethylsiloxane-Based PUr Elastomers Used for UV-Vis Spectroscopy}

Table A8. Film thicknesses of PSU films measured using a Mitutoyo Digimatic Thickness Gauge Series 547. Values are given as an average from three measurements.

\begin{tabular}{lcc}
\hline & PSU & Film Thickness $(\mathbf{m m})$ \\
\hline $\mathrm{H}_{12}$ MDI-1.6\% & 0.36 \\
$\mathrm{H}_{12}$ MDI-5\% & 0.33 \\
$\mathrm{H}_{12}$ MDI-10\% & 0.36 \\
$\mathrm{H}_{12}$ MDI-15\% & 0.34 \\
$\mathrm{H}_{12}$ MDI-20\% & 0.35 \\
IPDI-1.4\% & 0.34 \\
$\mathrm{IPDI}-5 \%$ & 0.35 \\
IPDI-10\% & $0.35-0.36$ \\
IPDI-15\% & $0.35-0.36$ \\
IPDI-20\% & 0.36 \\
MDI-1.5\% & 0.35 \\
CHDI-1.0 $\%$ & 0.36 \\
\hline
\end{tabular}




\section{References}

1. Huang, S.-L.; Chao, M.-S.; Ruaan, R.-C.; Lai, J.-Y. Microphase separated structure and protein adsorption of polyurethanes with butadiene soft segment. Eur. Polym. J. 2000, 36, 285-294. [CrossRef]

2. Kang, J.; Erdodi, G.; Brendel, C.M.; Ely, D.; Kennedy, J.P. Polyisobutylene-based polyurethanes. V. Oxidative-hydrolytic stability and biocompatibility. J. Polym. Sci. Part A Polym. Chem. 2010, 48, 2194-2203. [CrossRef]

3. Zdrahala, R.J.; Zdrahala, I.J. Biomedical Applications of Polyurethanes: A Review of Past Promises, Present Realities, and a Vibrant Future. J. Biomater. Appl. 1999, 14, 67-90. [CrossRef] [PubMed]

4. Gunatillake, P.A.; Meijs, G.F. Polyurethanes in Biomedical Engineering. In Encyclopedia of Materials: Science and Technology, 2nd ed.; Buschow, K.H.J., Cahn, R.W., Flemings, M.C., Ilschner, B., Kramer, E.J., Mahajan, S., Veyssiere, P., Eds.; Elsevier: Oxford, UK, 2001; pp. 7746-7752.

5. Wang, W.; Wang, C. Polyurethane for biomedical applications: A review of recent developments. In Design and Manufacture of Medical Devices; Davim, J.P., Ed.; Woodhead Publishing: Sawston, UK, 2012; pp. 115-151.

6. Ward, R.S.; Jones, R.L. Polyurethanes and Silicone Polyurethane Copolymers. In Comprehensive Biomaterials; Ducheyne, P., Ed.; Elsevier: Oxford, UK, 2011; pp. 431-477.

7. Simmons, A.; Padsalgikar, A.D.; Ferris, L.M.; Poole-Warren, L.A. Biostability and biological performance of a PDMS-based polyurethane for controlled drug release. Biomaterials 2008, 29, 2987-2995. [CrossRef] [PubMed]

8. Sivak, W.N.; Zhang, J.; Petoud, S.; Beckman, E.J. Simultaneous drug release at different rates from biodegradable polyurethane foams. Acta Biomater. 2009, 5, 2398-2408. [CrossRef]

9. Jiang, J.; Fu, Y.; Zhang, Q.; Zhan, X.; Chen, F. Novel amphiphilic poly(dimethylsiloxane) based polyurethane networks tethered with carboxybetaine and their combined antibacterial and anti-adhesive property. Appl. Surf. Sci. 2017, 412, 1-9. [CrossRef]

10. Nowatzki, P.J.; Koepsel, R.R.; Stoodley, P.; Min, K.; Harper, A.; Murata, H.; Donfack, J.; Hortelano, E.R.; Ehrlich, G.D.; Russell, A.J. Salicylic acid-releasing polyurethane acrylate polymers as anti-biofilm urological catheter coatings. Acta Biomater. 2012, 8, 1869-1880. [CrossRef]

11. Moura, L.I.; Dias, A.M.; Carvalho, E.; De Sousa, H.C. Recent advances on the development of wound dressings for diabetic foot ulcer treatment-A review. Acta Biomater. 2013, 9, 7093-7114. [CrossRef]

12. Duxbury, P.J.; Harvey, J.R. Systematic review of the effectiveness of polyurethane-coated compared with textured silicone implants in breast surgery. J. Plast. Reconstr. Aesthetic Surg. 2016, 69, 452-460. [CrossRef]

13. Bernacca, G.M.; Straub, I.; Wheatley, D.J. Mechanical and morphological study of biostable polyurethane heart valve leaflets explanted from sheep. J. Biomed. Mater. Res. 2002, 61, 138-145. [CrossRef]

14. Heijkants, R.G.J.C.; Van Calck, R.V.; De Groot, J.H.; Pennings, A.J.; Schouten, A.J.; Van Tienen, T.G.; Ramrattan, N.; Buma, P.; Veth, R.P.H. Design, synthesis and properties of a degradable polyurethane scaffold for meniscus regeneration. J. Mater. Sci. Mater. Med. 2004, 15, 423-427. [CrossRef] [PubMed]

15. Da Silva, G.R.; Junior, D.S.C.A.; Saliba, J.B.; Berdugo, M.; Goldenberg, B.T.; Naud, M.C.; Ayres, E.; Oréfice, R.L.; Cohen, F.B. Polyurethanes as supports for human retinal pigment epithelium cell growth. Int. J. Artif. Organs 2011, 34, 198-209. [CrossRef] [PubMed]

16. Gunatillake, P.A.; Martin, D.J.; Meijs, G.F.; McCarthy, S.J.; Adhikari, R. Designing biostable polyurethane elastomers for biomedical implants. Aust. J. Chem. 2003, 56, 545-557. [CrossRef]

17. Yang, J.; Gao, Y.; Li, J.; Ding, M.; Chen, F.; Tan, H.; Fu, Q. Synthesis and microphase separated structures of polydimethylsiloxane/ polycarbonate-based polyurethanes. RSC Adv. 2013, 3, 8291-8297. [CrossRef]

18. Adhikari, R.; Gunatillake, P.A.; McCarthy, S.J.; Meijs, G.F. Mixed macrodiol-based siloxane polyurethanes: Effect of the comacrodiol structure on properties and morphology. J. Appl. Polym. Sci. 2000, 78, 1071-1082. [CrossRef]

19. Martin, D.J.; Warren, L.A.; Gunatillake, P.A.; McCarthy, S.J.; Meijs, G.F.; Schindhelm, K. Polydimethylsiloxane/polyether-mixed macrodiol-based polyurethane elastomers: Biostability. Biomaterials 2000, 21, 1021-1029. [CrossRef]

20. CSIRO. Elast-Eon ${ }^{\mathrm{TM}}$ Biocompatible Polyurethane. 2008. Available online: https://csiropedia.csiro.au/elast-eon-biocompatiblepolyurethane/ (accessed on 1 August 2018.).

21. Yilgör, I.; Yilgör, E.; Wilkes, G.L. Critical parameters in designing segmented polyurethanes and their effect on morphology and properties: A comprehensive review. Polymer 2015, 58, A1-A36. [CrossRef]

22. Yilgor, E.; Eynur, T.; Bakan, M.; Yilgor, I.; Wilkes, G.L. Contribution of soft segment entanglement on the tensile properties of silicone-urea copolymers with low hard segment contents. Polymer 2009, 50, 4432-4437. [CrossRef]

23. Adhikari, R.; Gunatillake, P.A.; Meijs, G.F.; McCarthy, S.J. The effect of diisocyanate isomer composition on properties and morphology of polyurethanes based on 4,4'-dicyclohexyl methane diisocyanate and mixed macrodiols (PDMS-PHMO). J. Appl. Polym. Sci. 1999, 73, 573-582. [CrossRef]

24. Saralegi, A.; Exteberria, A.; Fernándes-d'Arlas, B.; Mondragon, I.; Eceiza, A.; Corcuera, M.A. Effect of $\mathrm{H}_{12} \mathrm{MDI}$ isomer composition on mechanical and physico-chemical properties of polyurethanes based on amorphous and semicrystalline soft segments. Polym. Bull. 2013, 70, 2193-2210. [CrossRef]

25. Joseph, M.D.; Savina, M.R.; Harris, R.F. Effects on properties of varying the cis/trans isomer distribution in polyurethane elastomers made with 1,4-cyclohexane diisocyanate. J. Appl. Polym. Sci. 1992, 44, 1125-1133. [CrossRef]

26. Prisacariu, C.; Buckley, C.P.; Caraculacu, A.A. Mechanical response of dibenzyl-based polyurethanes with diol chain extension. Polymer 2005, 46, 3884-3894. [CrossRef] 
27. Prisacariu, C.; Scortanu, E. Effect of Increasing the Hard Segment Percentage on the Mechanical Response of Selected Polyurethane Films. High Perform. Polym. 2010, 22, 876-887. [CrossRef]

28. Das, S.; Cox, D.F.; Wilkes, G.L.; Klinedinst, D.B.; Yilgor, I.; Yilgor, E.; Beyer, F.L. Effect of Symmetry and H-bond Strength of Hard Segments on the Structure-Property Relationships of Segmented, Nonchain Extended Polyurethanes and Polyureas. J. Macromol. Sci. Part B 2007, 46, 853-875. [CrossRef]

29. Yilgor, I.; Yilgor, E. Structure-Morphology-Property Behavior of Segmented Thermoplastic Polyurethanes and Polyureas Prepared without Chain Extenders. Polym. Rev. 2007, 47, 487-510. [CrossRef]

30. Sami, S.; Yildirim, E.; Yurtsever, M.; Yurtsever, E.; Yilgor, E.; Yilgor, I.; Wilkes, G.L. Understanding the influence of hydrogen bonding and diisocyanate symmetry on the morphology and properties of segmented polyurethanes and polyureas: Computational and experimental study. Polymer 2014, 55, 4563-4576. [CrossRef]

31. Yildirim, E.; Yurtsever, M. The role of diisocyanate and soft segment on the intersegmental interactions in urethane and urea based segmented copolymers: A DFT study. Comput. Theor. Chem. 2014, 1035, 28-38. [CrossRef]

32. Riehle, N.; Thude, S.; Götz, T.; Kandelbauer, A.; Thanos, S.; Tovar, G.E.; Lorenz, G. Influence of PDMS molecular weight on transparency and mechanical properties of soft polysiloxane-urea-elastomers for intraocular lens application. Eur. Polym. J. 2018, 101, 190-201. [CrossRef]

33. Riehle, N.; Maurus, M.A.; Tovar, G.E.M.; Lorenz, G. Data on the synthesis of trans-1,4-cyclohexane diisocyanate from 1,4cyclohexane dicarboxylic acid. Data Brief. prepared for publication.

34. Riehle, N.; Götz, T.; Kandelbauer, A.; Tovar, G.E.M.; Lorenz, G. Data on the synthesis and mechanical characterization of polysiloxane-based urea-elastomers prepared from amino-terminated polydimethylsiloxanes and polydimethyl-methyl-phenylsiloxane-copolymers. Data Brief 2018, 18, 1784-1794. [CrossRef]

35. Riehle, N.; Thude, S.; Kandelbauer, A.; Tovar, G.E.M.; Lorenz, G. Synthesis of Soft Polysiloxane-urea Elastomers for Intraocular Lens Application. J. Vis. Exp. 2019, 145, e58590. [CrossRef] [PubMed]

36. Deutsches Institut für Normung e.V. Prüfung von Kautschuk und Elastomeren-Bestimmung von Reißfestigkeit, Zugfestigkeit, Reißdehnung und Spannungswerten im Zugversuch; Beuth Verlag GmbH: Berlin, Germany, 2017. (In German)

37. Deutsches Institut für Normung e.V. Biologische Beurteilung von Medizinprodukten-Teil 5: Prüfungen Auf In-vitro-Zytotoxizität; Beuth Verlag GmbH: Berlin, Germany, 2009. (In German)

38. Yılgör, E.; Yılgör, İ. Hydrogen bonding: A critical parameter in designing silicone copolymers. Polymer 2001, 42, 7953-7959. [CrossRef]

39. Barton, A.F.M. CRC Handbook of Solubility Parameters and Other Cohesion Parameters, 2nd ed.; CRC Press: Boca Raton, FL, USA, 1991.

40. Versteegen, R.M.; Sijbesma, R.P.; Meijer, E.W. Synthesis and Characterization of Segmented Copoly(ether urea)s with Uniform Hard Segments. Macromolecules 2005, 38, 3176-3184. [CrossRef]

41. Prisacariu, C. Polyurethane Elastomers-From Morphology to Mechanical Aspects; Springer: Vienna, Austria, 2011.

42. Dearth, R.; Mertes, H.; Jacobs, P. An overview of the structure/property relationship of coatings based on 4,4'-dicyclohexylmethane diisocyanate (H12MDI). Prog. Org. Coat. 1996, 29, 73-79. [CrossRef]

43. Hatada, K.; Ute, K.; Oka, K.I.; Pappas, S.P. Unambiguous 13C-NMR assignments for isocyanate carbons of isophorone diisocyanate and reactivity of isocyanate groups in Z- and E-stereoisomers. J. Polym. Sci. Part A Polym. Chem. 1990, 28, 3019-3027. [CrossRef]

44. Cionni, R.J. Intraocular lenses to restore and preserve vision following cataract surgery. In Essentials in Ophthalmology-Cataract and Refractive Surgery; Kohnen, T., Koch, D.D., Eds.; Springer: Berlin/Heidelberg, Germany, 2006; pp. 3-9.

45. Yilgör, E.; Burgaz, E.; Yurtsever, E.; Yilgör, I. Comparison of hydrogen bonding in polydimethylsiloxane and polyether based urethane and urea copolymers. Polymer 2000, 41, 849-857. [CrossRef]

46. Bozukova, D.; Pagnoulle, C.; Jérôme, R.; Jérôme, C. Polymers in modern ophthalmic implants-Historical background and recent advances. Mater. Sci. Eng. R Rep. 2010, 69, 63-83. [CrossRef]

47. Rosu, D.; Rosu, L.; Cascaval, C.N. IR-change and yellowing of polyurethane as a result of UV irradiation. Polym. Degrad. Stab. 2009, 94, 591-596. [CrossRef]

48. Versteegen, R.M.; Kleppinger, R.R.; Sijbesma, A.R.P.; Meijer, E.B. Properties and Morphology of Segmented Copoly(ether urea)s with Uniform Hard Segments. Macromolecules 2006, 39, 772-783. [CrossRef]

49. Arun, A.; Baack, K.K.; Gaymans, R. Polyurethane tri-block copolymers-Synthesis, mechanical, elastic, and rheological properties. Polym. Eng. Sci. 2009, 50, 747-755. [CrossRef]

50. De, D.; Gaymans, R. Thermoplastic Polyurethanes with TDI-Based Monodisperse Hard Segments. Macromol. Mater. Eng. 2009, 294, 405-413. [CrossRef]

51. Wittbecker, E.L.; Houtz, R.C.; Watkins, W.W. Elastic N-Substituted Polyamides. Ind. Eng. Chem. 1948, 48, 875-879. [CrossRef]

52. Ertem, S.P.; Yilgor, E.; Kosak, C.; Wilkes, G.L.; Zhang, M.; Yilgor, I. Effect of soft segment molecular weight on tensile properties of poly(propylene oxide) based polyurethaneureas. Polymer 2012, 53, 4614-4622. [CrossRef]

53. Jewrajka, K.S.; Yilgor, E.; Yilgor, I.; Kennedy, J.P. Polyisobutylene-based segmented polyureas. I. Synthesis of hydrolytically and oxidatively stable polyureas. J. Polym. Sci. Part A Polym. Chem. 2009, 47, 38-48. [CrossRef]

54. Buckwalter, J.D.; Zhang, M.; Inglefield, D.L.; Moore, R.B.; Long, T.E. Synthesis and characterization of siloxane-containing poly(urea oxamide) segmented copolymers. Polymer 2013, 54, 4849-4857. [CrossRef] 
55. Buckwalter, D.J.; Inglefield, D.L.; Enokida, J.S.; Hudson, A.G.; Moore, R.B.; Long, T.E. Effects of Copolymer Structure on the Mechanical Properties of Poly(dimethyl siloxane) Poly(oxamide) Segmented Copolymers. Macromol. Chem. Phys. 2013, 214, 2073-2082. [CrossRef]

56. Yilgor, I.; Eynur, T.; Bilgin, S.; Yilgor, E.; Wilkes, G.L. Influence of soft segment molecular weight on the mechanical hysteresis and set behavior of silicone-urea copolymers with low hard segment contents. Polymer 2011, 52, 266-274. [CrossRef]

57. Hellgeth, J.W.; Ward, T.C. Mechanical hysteresis of a polyether polyurethane thermoplastic elastomer. Polym. Eng. Sci. 1993, 33, 1170-1176.

58. Caracciolo, P.C.; Buffa, F.; Abraham, G.A. Effect of the hard segment chemistry and structure on the thermal and mechanical properties of novel biomedical segmented poly(esterurethanes). J. Mater. Sci. Mater. Med. 2009, 20, 145-155. [CrossRef]

59. Yildirim, E.; Yurtsever, M.; Wilkes, G.L.; Yilgör, I. Effect of intersegmental interactions on the morphology of segmented polyurethanes with mixed soft segments: A coarse-grained simulation study. Polymer 2016, 90, 204-214. [CrossRef]

60. Kuo, A.C.M. Polydimethylsiloxanes. In Polymer Data Handbook; Mark, J.E., Ed.; Oxford University Press: Oxford, UK, 2009; pp. 411-435.

61. Adhikari, R.; Gunatillake, P.A.; McCarthy, S.J.; Meijs, G.F. Low-modulus siloxane-based polyurethanes. I. Effect of the chain extender 1,3-bis(4-hydroxybutyl)1,1,3,3-tetramethyldisiloxane (BHTD) on properties and morphology. J. Appl. Polym. Sci. 2001, 83, 736-746. [CrossRef]

62. Adhikari, R.; Gunatillake, P.A.; McCarthy, S.J.; Bown, M.; Meijs, G.F. Low-modulus siloxane-polyurethanes. Part II. Effect of chain extender structure on properties and morphology. J. Appl. Polym. Sci. 2003, 87, 1092-1100. [CrossRef]

63. Yildirim, E.; Yurtsever, M.; Yurtsever, E.; Yilgor, I.; Yilgor, E. Multiscale Modeling of the Morphology and Properties of Segmented Silicone-Urea Copolymers. J. Inorg. Organomet. Polym. Mater. 2012, 22, 604-616. [CrossRef]

64. Abouzahr, S.; Wilkes, G.; Ophir, Z. Structure-property behaviour of segmented polyether-MDI-butanediol based urethanes: Effect of composition ratio. Polymer 1982, 23, 1077-1086. [CrossRef]

65. Wang, C.B.; Cooper, S.L. Morphology and properties of segmented polyether polyurethane ureas. Macromolecules 1983, 16, 775-786. [CrossRef]

66. Neira-Velázques, M.G.; Rodríguez-Hernández, M.T.; Ernesto, H.-H.; Ruiz-Martínez, A.R.Y. Polymer Molecular Weight Measurement. In Handbook of Polymer Synthesis, Characterization, and Processing; John Wiley \& Sons: Hoboken, NJ, USA, 2013.

67. Gurunathan, T.; Mohanty, S.; Nayak, S.K. Isocyanate terminated castor oil-based polyurethane prepolymer: Synthesis and characterization. Prog. Org. Coat. 2015, 80, 39-48. [CrossRef]

68. Wachholz, S.; Keidel, F.; Just, U.; Geissler, H.; Käppler, K. Analysis of a mixture of linear and cyclic siloxanes by cryo-gas chromatography-Fourier transform infrared spectroscopy and gas chromatography-mass spectrometry. J. Chromatogr. A 1995, 693, 89-99. [CrossRef] 